\title{
Mécanique des terrains perméables
}

\section{The mechanics of permeable soils}

\author{
PAR J. FERRANDON
}

MAITRE DE GONFÉRENGES A L'ÉCOLE POLYTEGHNIQUE

\begin{abstract}
Le texte ci-dessous résume la matière d'une série de conférences faites aux Elèves Ingénieurs de l'Institut Polytechnique de Grenoble au cours de la session scolaire 1953-1954. Leur objet est l'examen de certaines propriétés des terrains perméables particulièrement utiles pour les applications.

La première partie expose les lois de l'écoulement laminaire en généralisant, par l'introduction du tenseur de perméabilité, la loi de Darcy aux milieux isotropes.

La seconde partie traite de l'équilibre limite des sols cohérents sans frottement interne et des sols pulvérulents par la méthode analytique de Cauchy, par laquelle les lignes de glissement apparaissent comme courbes caractéristiques d'un système d'équation aux dérivées partielles.

La troisième partie envisage les phénomènes de propagation de discontinuités dans un sol perméable, compte tenu de l'existence de denx phases solide et liquide en présence.

Enfin une note de $M$. $F$. Serre sur l'évolution en fonction $d u$ temps du tassement des couches argileuses aborde le problème du tassement des fondations d'une manière tout d̀ fait générale.
\end{abstract}

\begin{abstract}
The following text is a resumé of a series of lectures delivered to engineering students at the Grenoble Polytechnic Institute during the 1953-1954 session. The object of the lectures was to examine those properties of permeable soils which are of special importance in practical problems.

The first part treats the laws of laminar flow. generalising Darcy's law for isotropic soils by introducing the permeability tensor.

The second part deals with the limiting equilibrium of cohesive soils without internal friction, and of cohesionless soils. The analytical method of Cauchy is used and gives the failure lines as characteristic curves of a system of partial differential equations.

The third part considers the phenomena of propagation of discontinuities in a permeable soil taking into account the existence of both solid and liquid stages.

Finally a note by M. F. SERRE on the time element in the settlement of clay strata introduces the problem of foundation settlement in a very general manner.
\end{abstract}

\section{ETATS LIMITES DES MASSIFS FILTRANTS}

Les massifs naturels (sables, argiles, vases) sur lesquels sont édifiés les constructions sont, en plus des charges superficielles que leur transmettent les fondations de ces derniers, sollicités par des forces de volume comportant leur poids propre et les actions, mises en évidence précédemment, de l'eau y filtrant.

La seconde partie de cet exposé est consacrée à l'examen des conditions d'équilibre de ces sols sous les forces précitées.

Il y sera traité successivement :

- de l'état local des contraintes,

- de l'état d'équilibre limite des sols cohérents à frottement nul,

- de l'état d'équilibre limite des sols pulvérulents.

(*) La Houille Blanche, $\mathrm{n}^{\circ} 4,1954, \mathrm{pp} .466-480$.
Le premier point est, à dire vrai, classique, mais sa connaissance est indispensable pour la compréhension de la suile. Au deuxième s'attachent des équations intégrables d'où procèdent des résultats tangibles immédiatement utilisables par l'ingénieur. Il en est autrement du dernier pour lequel il faudra se contenter de généralités et de quelques résultats partiels d'ailleurs fructueux.

\section{I}

\section{ETUDE LOCALE DES CONTRAINTES}

\section{Continuité macroscopique.}

Les sols pulvérulents ou cohérents formés par la juxtaposition d'éléments de nature, de forme, et de dimensions très diverses, ne sont pas sus- 
ceptibles d'une analyse précise faisant étal de leur constitution interne complexe. Mais la plupart des observations et investigations d'où procèdent les résultats utiles aux ingénieurs, concernent des extensions finies d'un nombre si élevé de particules qu'il apparaît légitime de les traiter comme des continus macroscopiques eu égard à la recherche des grandeurs moyennes caractéristiques de leur état mécanique.

\section{REPRÉSENTAtion PLANE DE LA DISTRIBUTION LOCALE DES CONTRAINTES.}

Il est ainsi possible de définir pour les massif's en terre des tensions internes ou contraintes analogues à celles de la mécanique des continus matériels déformables et en possédant toutes les propriétés géométriques supposées connues du lecteur. La possibilité d'une représentation plane de l'état des forces intérieures est l'une de celles qui sera souvent prise en considération par la suite et dont il convient, afin de fixer, une fois pour toutes, certaines conventions et notations, de rappeler successivement les développements essentiels.

Soit à cet effet un massif continu, supposé tout d'abord bidimensionnel. En tout point $\mathrm{M}$, les axes rectangulaires de coordonnées étant de directions quelconques, et les compressions étant comptées positivement, soient $\mathrm{N}_{1}, \mathrm{~N}_{2}, \mathrm{~T}_{3}$ (notations de Lamé) les coordonnées du tenseur des contraintes. Les composantes normales $\mathbf{N}$ et tangentielles $\mathrm{T}$ de la contrainte relative à l'élément de droite (P) passant par $M$ et défini par l'angle $\theta$ de sa normale $M n$ et de $O x$ sont :

$\left\{\begin{array}{l}\mathrm{N}=\frac{\mathrm{N}_{1}+\mathrm{N}_{2}}{2}+\frac{\mathrm{N}_{1}-\mathrm{N}_{2}}{2} \cos 20+\mathrm{T}_{3} \sin 20 \\ \mathrm{~T}=-\frac{\mathrm{N}_{1}-\mathrm{N}_{2}}{2} \sin 20+\mathrm{T}_{3} \cos 20\end{array}\right.$

Dans le système d'axes rectangulaires liés à (P), Mn, Mt (fig. 13), le point $m(\mathrm{~N}, \mathrm{~T})$ décril ainsi, lorsque $\theta$ rarie, le cercle $(\Gamma)$ passant par les
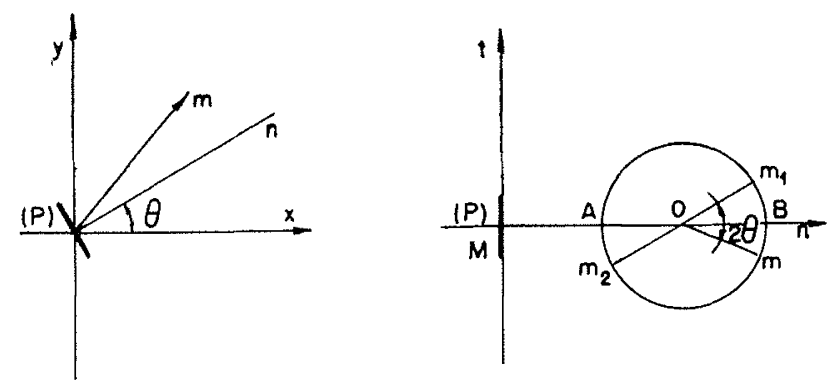

Fis. 13

points $m_{1}\left(\mathbf{N}_{1}, \mathrm{~T}_{3}\right)$ et $m_{2}\left(\mathrm{~N}_{2},-\mathrm{T}_{3}\right)$ correspondanl respectivement à $0=0$ et $0=(\pi / 2)$, et diamétra- lement opposés. Le centre $O$ de (T) a pour abscisse $\omega=(1 / 2)\left(N_{1}+N_{2}\right)$. L'angle $\left(m_{1}\right.$ om $)$ est égal à $2 \theta$. Les contraintes principales ont pour grandeurs les abscisses $a$ et $b$ des points $A$ et $B$ d'intersection de $(\Gamma)$ et de $m n$; elles font avec $\mathrm{M} x$ les angles (1/2) $\left(\mathrm{om}_{1} \mathrm{~A}\right)$ et $(1 / 2)\left(o m_{1} \mathrm{~B}\right)$, étant entendu que les angles, dans le plan $n \mathrm{M} t$ de la représentation, sont comptés positivement dans le sens amenant Mt sur Mn .

L'extension de la correspondance ci-dessus aux massifs tridimensionnels résulte du théorème suivant dont est seulement donné l'énoncé. En un point quelconque $M$ du massif, soit $\bar{\Theta}$ la contrainte relative à l'élément plan $(P)$ de normale $\mathrm{M} n$. Sur Mn portons les trois points A, B, C (fig. 14) dont les abscisses par rapport à l'ori-
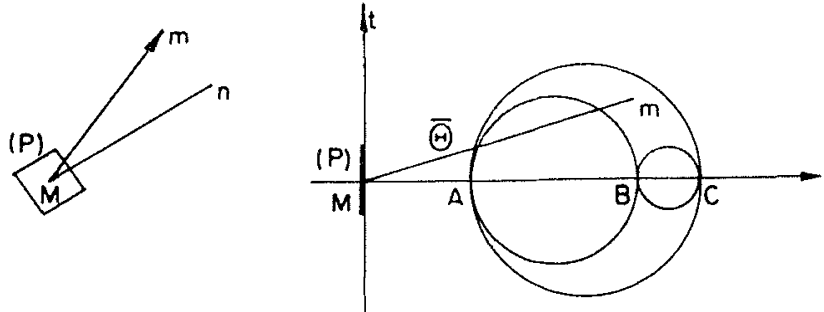

Fin. 14

gine $\mathrm{M}$ sont les valeurs $a, b, c$ des contraintes principales en $\mathrm{M}$; puis, dans le plan $\mathrm{Mn} \bar{\Theta}$, tracons les trois cercles de diamètre $\mathrm{AB}, \mathrm{BC}, \mathrm{CA}$. L'extrémité $m$ de la contrainte $\bar{\Theta}$ est, quelle que soit la position de $\mathrm{Mn}$, intérieure au plus grand des cercles et extérieure aux deux autres.

\section{Frottement interne et cohésion. Lor DF Coulomb.}

L'expérience conduit à admettre que toute rupture d'équilibre d'un massif en terre, sous l'action de sollicitations extérieures particulières, s'amorce par des glissements irréversibles le long de certains de ses éléments de surface. En un point $M$, soit (P) l'un d'eux de normale $M n$ ef $\Theta(N, T)$ la contrainte relative à celui-ci. La condition de non-apparition des désordres en cause consiste en une inégalité de la forme :

$$
|\mathrm{T}|<\mathrm{N} \operatorname{tg} \varphi+\mathrm{C}
$$

traduisant la loi de Covlomb.

En toute généralité, position et de l'orientation de Mn. Dans l'hypothèse, très généralement adoptée par la suite, d'un sol isotrope, ces paramètres sont, en un point donné, des paramètres désignant respeclivement l'angle de frottement interne et la cohésion en ce point. Si, par surcroît, le massif est homogène, ces quantités sont des constantes ab- 
solues permettant, du point de vue de la stabilité interne, de le caractériser.

La connaissance de la valeur des coefficients caractéristiques $\varphi$ et C qui s'avère ainsi essentielle est demandée à l'expérience dans chaque cas particulier. Sont donnés, à titre indicatif, dans le tableau ci-dessous, quelques ordres de grandeur.

\begin{tabular}{|c|c|c|}
\hline \multicolumn{3}{|c|}{$\begin{array}{c}\text { Valeurs moyennes et approximatives des coefficients } \\
\text { caractéristiques de divers terrains }\end{array}$} \\
\hline Désignation du sol & $\begin{array}{c}\text { Angle de } \\
\text { frottement interne } \\
\text { (en degrés) }\end{array}$ & $\begin{array}{c}\text { Cohésion } \\
\mathrm{C} \\
\text { hectopièzes }\end{array}$ \\
\hline 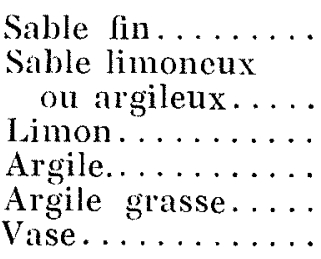 & $\begin{array}{rll}33 & \text { à } & 35 \\
27 & \text { à } & 33 \\
25 & \text { à } & 33 \\
25 & \text { à } & 27 \\
15 & \text { à } & 25 \\
0 & \text { à } & 15\end{array}$ & $\begin{array}{lll}0,5 & \text { à } & 1 \\
0,2 & \text { à } & 0,5 \\
0,1 & \text { à } & 0,2\end{array}$ \\
\hline
\end{tabular}

Les matériaux pulvérulents ou à cohésion nulle comprennent les sables, les matériaux sans frottement interne, certaines vases et notamment la plupart des vases marines gorgées d'eau. Entre ces deux catégories extrêmes s'étend la gamme des argiles et des marnes.

II y a lieu de noter le caractère approché de la loi de Covlomb. Celle-ci assigne en effel aux divers sols qu'elle concerne une courbe intrinsèque formée de deux droites symétriques par rapport à l'axe des contraintes normales, et cette forme n'est manifestement pas compatible avec la propriété de ceux-ci de se rompre sous des charges de traction relativement modérées. En fait, si les courbes intrinsèques des sables (fig. 15) se réduisent à deux demi-droites du plan

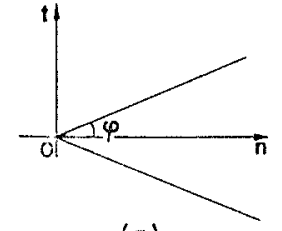

(a)

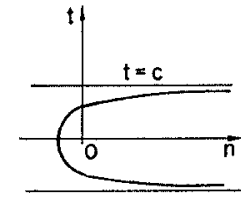

(b)

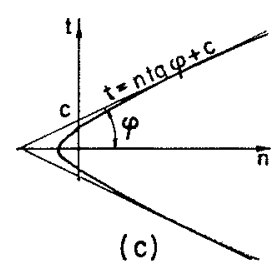

Firi, 15 par rapport à l'axe des $n$, et avec lesquelles elles peuvent légitimement être confondues pour les compressions quelque peu importantes. Dans cette éventualité, un massif isotrope sera donc stable si, en chacun de ses points M, la condition (2) est satisfaite pour toute direction $\mathrm{Mn}$, autrement dit, si le cercie représentatif des contraintes les plus dangereuses est situé au-dessous de la droite d'équation :

$$
t=n \operatorname{tg} \varphi+\mathrm{C}
$$

\section{Notion D'Éguilibre limite.}

Il s'agit d'étudier, dans une circonstance très particulière qui sera généralisée ultérieurement, les propriétés essentielles d'un massif en terre en équilibre limite, c'est-à-dire tel que certaines modifications très petites des sollicitations extérieures y provoquent dans une zone finie des désordres irréversibles. En tout point d'un tel massif, sous les réserves énoncées ci-dessus, quant à l'intensité des pressions, est alor's satisfaite l'égalité de Coulomb. Est donc très limitativement envisagé un massif pulvérulent (sable sec) de poids spécifique $\Delta$ et d'angle de frottement interne $\varphi$, limité vers le haut par un plan hori-

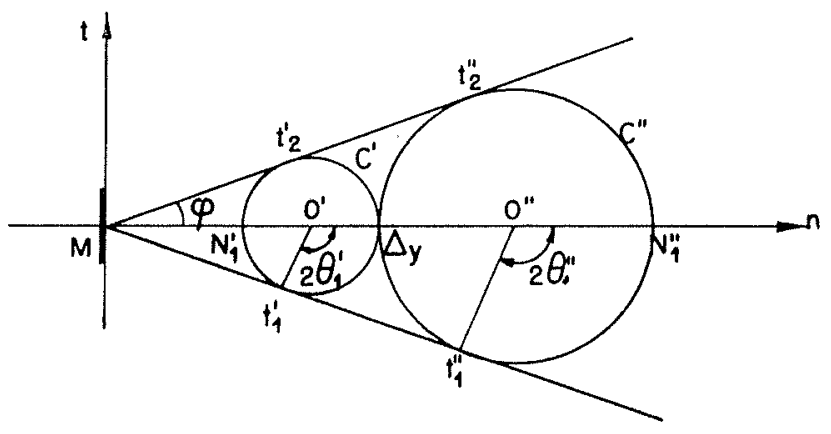

Fia. 16

zontal non chargé. Ses éléments horizonlaux sont principaux pour la distribution des contraintes qui y règnent, et à la profondeur $y$, l'intensité de la pression verticale est $\Delta y$. La considération de l'équilibre du massif ne suffit pas à déterminer la pression principale horizontale $\mathrm{N}_{1}$ qui peut être comprise entre deux valeurs extrêmes $N^{\prime}{ }_{1}$ et $\mathrm{N}^{\prime \prime}{ }_{1}$, abscisses des points d'intersection de $\mathrm{Mn}$ et des cercles d'équilibres limites $\mathrm{C}^{\prime}$ et $\mathrm{C}^{\prime \prime}$ passant par le point d'abscisse $\Delta y$ de cet axe et tangents à la droite de Coulomi : $t=n \operatorname{tg} \varphi$ (fig. 16). Tous les états possibles sont ainsi compris entre l'état d'équilibre inférieur $\mathrm{N}_{1}^{\prime}$ ou de poussée, et l'état d'équilibre supérieur $\mathrm{N}^{\prime \prime}{ }_{1}$ ou de butée sur lesquels va précisément porter notre attention.

Dans chacun de ceux-ci, à condition d'attri- $n>o$ passant par l'origine et inclinées de $\pm \varphi$ (angle de frottement interne) sur l'axe des $n$, celles des vases. (fig. $15 b$ ) et celles des argiles ou des marnes (fig. 15 c) sont curvilignes et admettent respectivement pour asymptotes les faisceaux de droites $t= \pm \mathrm{C}, t= \pm n \operatorname{tg} \varphi+\mathrm{C}$ dont elles possèdent naturellement la symétrie 
buer à la constante A l'une ou l'autre des valeurs résultant directement de la figure :

$$
\mathrm{A}^{\prime}=\Delta \operatorname{tg}^{2}\left(\frac{\pi}{4}-\frac{\varphi}{2}\right) ; \mathrm{A}^{\prime \prime}=\Delta \operatorname{tg}^{2}\left(\frac{\pi}{4}+\frac{\varphi}{2}\right)
$$

et qui caractérisent l'état de poussée et l'état de butée respectivement, les contraintes en tout point résultent des expressions :

$$
\mathrm{N}_{1}=\Delta y, \quad \mathrm{~N}_{2}=\Delta y, \quad \mathrm{~T}_{3}=0 .
$$

Considérons l'état de poussée. En chaque point $M$ du massif, sur les deux éléments $e_{1}^{\prime}$ et $e_{2}^{\prime}$ d'images $t_{1}^{\prime}$ et $t_{2}^{\prime}$, la contrainte présente avec la normale $M n$ l'inclinaison maximum ( $\phi$ dans le cas actuel d'un sol pulvérulent), symétriques par rapport à l'horizon $o x$, ils sont inclinés sur cet axe des angles (fig. 17) :

$$
0_{1}^{\prime}=\frac{\pi}{4}+\frac{\varphi}{2}, \quad \theta_{2}^{\prime}=-\left(\frac{\pi}{4}+\frac{\varphi}{2}\right) .
$$

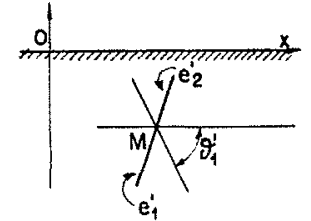

(a)

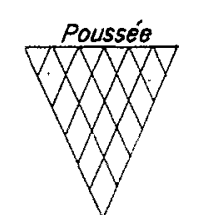

(b)

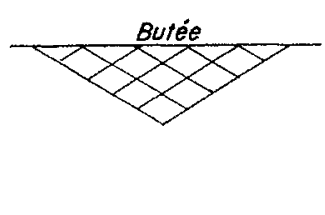

(c)
Fig. 17

L'ensemble des $e_{1}^{\prime}$ ou éléments de glissement de première espèce est porté par le réseau des droites de glissement de première espéce distribuées en faisceau de parallèles symétriques par rapport à $o x$ (fig. 17 b). L'état de butée comporte des résultats analogues. Aux éléments $e^{\prime \prime}{ }_{1}$ et $e^{\prime \prime}{ }_{2}$ d'images $t^{\prime \prime}{ }_{1}$ et $t^{\prime \prime}{ }_{2}$ (fig. 16) correspondent de même les lignes de glissement de seconde espèce faisant avec ox les angles :

$$
\theta^{\prime \prime}{ }_{1}=\frac{\pi}{4}-\frac{\varphi}{2}, \quad \theta^{\prime \prime}=\cdots\left(\frac{\pi}{4}-\frac{\varphi}{2}\right),
$$

et se groupant en faisceaux parallèles (fig 17 c).

Remarque: Les équilibres limites très particuliers donnant lieu, tels ceux décrits ci-dessus, à lignes de glissement parallèles, sont dits équilibres de Ranisine.

\section{Structure hécanigue des massifs en ÉQUi- LIBRE.}

Afin de justifier les développements qui vont suivre et d'en faciliter la compréhension, quelques indications sur la construction des massifs en terre en équilibre sont utiles. Un tel ensemble est formé de zones de deux natures. Dans les premières $(\mathbf{E})$, justiciables en première approxi- mation de la théorie de l'élasticité, l'équilibre est surabondant, et une petite modification de l'état des contraintes résultant de la mise en jeu de forces accidentelles n'y déterminent que des perturbations quasi-réversibles. Le cercle représentatif des contraintes les plus dangereuses est en tout point situé au-dessous de la droite de CouLомв, $t=n \operatorname{tg} \varphi+$ C. Dans les secondes (L) en équilibre limite, inférieur ou supérieur, l'égalité $t=n \operatorname{tg} \varphi+\mathrm{C}$ est en tout point vérifiée. Le cercle représentatif des contraintes est tangent à la droite de Coulomb. Les régions ( $L$ ), qui peuvent d'ailleurs s'évanouir en arcs ou en points isolés où est atteint l'équilibre limite, sont séparées des régions (E) par des surfaces de glissements, intérieurs au massif ou constituant tout ou partie de sa frontière. Dans l'éventualité de la distribution plane des massifs horizontalement indéfinis, les directrices des surfaces de glissement cylindriques constituent des lignes de glissement.

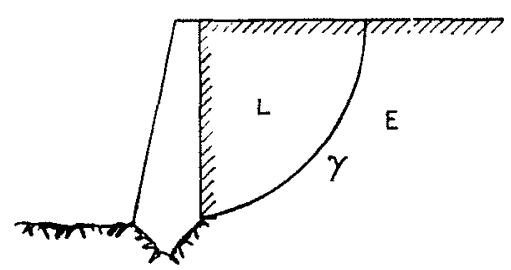

FIG. 18

Les circonstances très générales sont illustrées par deux exemples. La figure 18 est la coupe en travers d'un massif sur le point de céder retenu par un soutènement. On y a tracé la ligne de glissement $\gamma$ partant du pied du mur et séparant les zones $(\mathrm{E})$ et $(\boldsymbol{L})$.

La figure 19 est relative à un pieu transmettant au sol sous-jacent la charge $\overrightarrow{\mathbf{P}}$ qu'il reçoil en tête. Deux éventualités sont à distinguer : ou

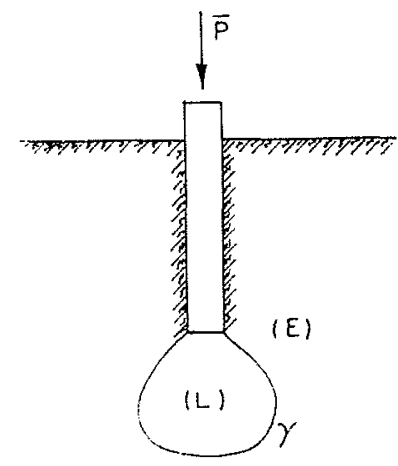

Fig. 19

bien la surface inférieure du pieu constitue la seule zone en équilibre limite, ou bien cet état s'établit dans une zone finie entourant cette extrémité et séparée par la ligne de glissement du reste $d u$ massif. 
L'expression ligne de glissement est susceptible de suggérer une interprétation erronée du processus de rupture qu'il convient de signaler. Pour les sols pulvérulents, nous verrons en effet que, sur chaque ligne de glissement, la contrainte fait avec la normale l'angle $\varphi$ de frottement interne. Il est alors tentant d'imaginer la rupture comme un glissement des zones (L) sur les zones (E) sans déformation à l'intérieur de ces régions respectives. Mais ce type de mouvement n'est cinématiquement possible que si les lignes de glissement se réduisent à des arcs de cercles ou à des segments de droites. Il résultera d'une analyse ultérieure que leur réseau est loin d'offrir une telle simplicité. En fait, l'observation des glissements de talus, par exemple, montre qu'entre deux zones en mouvement relatif apparemment indéformées, prennent naissance des zones troublées de singularités telles que failles, déchirement, y rendant illusoire toute investigation quantitative.

\section{II}

\section{THEORIE DE L'EQUILIBRE LIMITE DES SOLS COHERENTS SANS FROTTEMENT INTERNE}

1.

Plusieurs raisons militent en faveur d'une étude détaillée des états limites des sols dépourvus de frottement interne, dont certaines vases marines constituent un exemple d'une vaste extension :

- Leur considération conduit à des problèmes très généralement intégrables dont les solutions serviront de guides qualitatifs lors de circonstances moins favorables aux développements analytiques;

- Les résultats concernant les sols pulvérulents ne peuvent leur être étendus par le jeu des théorèmes des états correspondants $\left(^{*}\right)$ dont l'énoncé s'avère illusoire en ce qui les concerne; force est donc de procéder à une analyse directe;

(*) Théorème des états correspondants (CAQuor): Un système cohérent $(\varphi, C)$ est en équilibre si l'on peut lui faire correspondre un milieu pulvérulent en équilibre, occupant le même espace, admettant le même frottement apparent interne $(\varphi)$ et soumis, sur les parois qui le limitent, aux mêmes efforts que le milieu cohérent, augmenté de la pression hydrostatique :

$$
\mathbf{H}=-\mathrm{C} \operatorname{cotg} \varphi
$$

- Enfin, lors des ruptures de massifs gorgés d'eau, il semble bien que le frottenient, entre grains n'ayant en cette circonstance que des contacts fortuits d'importance négligeable, est nul ou quasi-nul, et que subsiste la seule cohésion comme conséquence d'attractions internes.

En vue d'une telle analyse, le sol est supposé soumis à des actions superficielles qui seront éventuellement précisées ultérieurement et à des forces de volume comportant les forces gravifiques et les forces hydrodynamiques de résultante :

$$
\overline{d F}=-\operatorname{grad}\{\bar{\Phi} \Phi+[\Delta-\varpi(1-m)] \gamma\} d \vartheta
$$

$(\mathrm{O} \gamma$, verticale ascendante), sur toute particule macroscopique de volume $d v$ 1.3.4.

Désignant par :

$$
\delta=\Delta-\bar{\omega}(1-m)^{(\star)}
$$

le poids spécifique du sol, compte tenu de la poussée archimédienne, et par :

$$
\Upsilon=\bar{\Phi}+\delta \gamma,
$$

le potentiel total dont les forces de volume dérivent, il vient indifféremment :

$$
\overline{d \mathrm{~F}}=-\operatorname{grad}(\varpi \Phi+\delta \gamma) d \vartheta=-\operatorname{grad} \gamma d \vartheta
$$

et les forces par unité de volume particulaire ont pour projections sur les axes : :

$$
\mathrm{X}=-\frac{\partial \mathrm{r}}{\partial \boldsymbol{x}}, \quad \mathrm{Y}=-\frac{\partial \mathrm{r}}{\partial y}, \quad \mathrm{z}=-\frac{\partial \mathrm{r}}{\partial z}
$$

\section{EQUATIONS INDĚFINIES D'ÉQUILIBRE LIMITE.}

Est envisagé limitativement un massif dépourvu de frottement interne, de cohésion $\mathrm{C}$, rapporté aux axes rectangulaires $\mathrm{O} x, \mathrm{O} y$ (verticale ascendante), de poids spécifique $\Delta$, sollicité par des forces de volume de l'espèce décrite cidessus. Dans cette hypothèse et en dehors des zones d'apparition de rupture par traction, est défini comme état limite celui pour lequel le cercle $(C)$ représentatif des contraintes (fig. 20) a pour rayon la valeur $\mathrm{C}$ de la cohésion, et se retrouve par suite tangent aux droites $t= \pm \mathrm{C}$ aux points $t_{1}$ et $t_{2}$, images des éléments pour lesquels l'inclinaison de la contrainte sur la nor-

(*) $\Delta$ désigne le poids spécifique apparent de la phase solide contenu dans le volume $d V$ dont le poids total est $\Delta d \eta^{*}$. 
male Mn est maximum. Ces éléments, dits de glissement, sont orthogonaux. Les lignes de glissement qui leur sont tangentes constituent ainsi un réseau réel de deux familles distinctes $\left(\Gamma_{1}\right)$

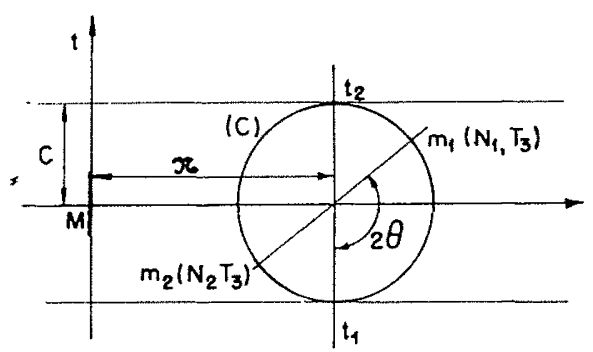

FIG. 20

et $\left(\mathrm{I}_{2}\right)$, d'ares $\mathrm{S}_{1}$ et $\mathrm{S}_{2}$. Par tout point du massif passent deux telles courbes orthogonales. Soient $\theta$ (fig. 21) l'angle de la direction $\left(\Gamma_{1}\right)$ avec la verticale $\mathrm{M} y$ et $o r$ la pression moyenne en $\mathrm{M}$, ou demi-somme des pressions principales, $\mathrm{N}_{1}, \mathrm{~N}_{2}, \mathrm{~T}_{3}$,

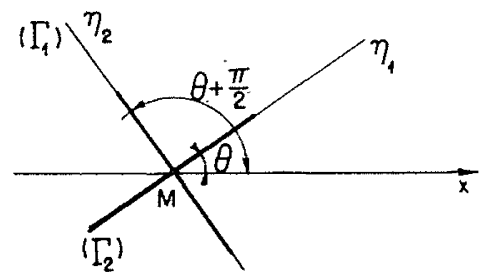

Fig. 21

les coordonnées du tenseur des contraintes relatif au point M. Compte tenu de l'égalité évidente :

$$
\mathscr{T}=1 / 2\left(\mathrm{~N}_{1}+\mathrm{N}_{2}\right)
$$

par la considération de la figure (1), il vient les relations :

$$
\left\{\begin{array}{l}
\mathrm{N}_{1}=x+\mathrm{C} \sin 20 \\
\mathrm{~N}_{2}=x-\mathrm{C} \sin 20 \\
\mathrm{~T}_{3}=-\mathrm{C} \cos 20
\end{array}\right.
$$

définissant les composantes de Lamé propres à l'équilibre limite, en fonction des deux paramètres $g r$ et $\theta$. Par l'introduction de ceux-ci, les cquations indéfinies de l'équilibre :

$$
\left\{\begin{array}{l}
\frac{\partial \mathrm{N}_{1}}{\partial x}+\frac{\partial \mathrm{T}_{3}}{\partial y}+\frac{\partial \gamma^{*}}{\partial x}=0 \\
\frac{\partial \mathrm{T}_{3}}{\partial x}+\frac{\partial \mathrm{N}_{2}}{\partial y}+\frac{\partial \gamma^{\prime}}{\partial y}=0
\end{array}\right.
$$

en posant :

$$
\pi=9 r+r
$$

s'écrivent :

$\left\{\begin{array}{l}\frac{\partial \pi}{\partial x}+2 C \cos 2 \theta \frac{\partial \theta}{\partial x}+2 C \sin 2 \theta \frac{\partial \theta}{\partial y}=0 \\ \frac{\partial \pi}{\partial y}-2 C \cos 2 \theta \frac{\partial \theta}{\partial y}+2 C \sin 2 \theta \frac{\partial \theta}{\partial x}=0 .\end{array}\right.$

Elles constituent un système de deux équations aux dérivées partielles de $\pi$ et $\theta$, dont va être entreprise la discussion.

\section{LE PRoblème de CAUChy POUR LES Fonctions $\pi$ (OU $\vartheta$ ) ET $\theta$.}

Cherchons à quelles conditions est possible la détermination de deux fonctions $\pi$ et $\theta$, solutions du système (6) prenant sur un $\operatorname{arc} \gamma(\xi, \eta)$ apparlenant au massif des valeurs $\pi(\xi, \eta), \theta(\xi, \eta)$ résultant d'une distribution donnée des contraintes le long de $\gamma$. Est ainsi posé le problème de CAUCHY pour les équations (6). Celles-ci, écrites pour $x=\xi, y=n$ et les relations :

$d \pi=\frac{\partial \pi}{\partial \xi} d \xi+\frac{\partial \pi}{\partial \eta}, \quad d \theta=\frac{\partial \theta}{\partial \xi} d \xi+\frac{\partial \theta}{\partial \eta} d \eta$

forment, par rapport aux dérivées partielles des fonctions $\pi$ et 0 , un système linéaire de quatre équations à quatre inconnues dont le déterminant est :

$$
\mathrm{D}=\left|\begin{array}{cccc}
1 & 0 & 2 \mathrm{C} \cos 2 \theta & 2 \mathrm{C} \sin 2 \theta \\
0 & 1 & 2 \mathrm{C} \sin 2 \theta & -2 \mathrm{C} \cos 2 \theta \\
d \xi & d r_{1} & 0 & 0 \\
0 & 0 & d \xi & d \eta
\end{array}\right|
$$$$
=2 \mathrm{C}(\cos \theta d \xi+\sin \theta d \eta)\left(-\sin \theta d \xi+\cos \theta d \eta_{1}\right)
$$

Si D est différent de zéro sur ( $\gamma)$, la résolution des équations est possible sur cette ligne. Par différenciations successives on peut alors calculer des dérivées de tous ordres de $\pi$ et 0 et construire les développements tayloriens de ces fonctions au roisinage de $\%$.

L'annulation de $\mathrm{D}$, entraìnant une impossibililé ou une indétermination pour la résolution en question se produit, si l'arc $\gamma$ se confond avec une ligne de glissement. Si celle-ci est de première espèce (famille $\Gamma_{1}$ ):

$$
\cos \theta d \xi+\sin \theta d \eta=0
$$
$\left.\Gamma_{2}\right):$

Si c'est une ligne de seconde espèce (famille

$$
\cos \left(0+\frac{\pi}{2}\right) d \xi+\sin \left(\theta+\frac{\pi}{2}\right) d \eta=0
$$

On en conclut que la résolution du problème 
de Cauchy est généralement impossible à partir des données arbitraires $\pi(\xi, \eta), \theta(\xi, \eta)$ relatives à une ligne de glissement quelconque du massif. Pour qu'elle soit indéterminée doit exister en ouLre, entre les donnćes $\pi$ et 0 et pour chacune des familles $\left(\Gamma_{1}\right)$ et $\left(\Gamma_{2}\right)$, une relation différentielle que nous allons former $\left({ }^{\star}\right)$. A cet effet, multiplions la première équation (6) par $d \xi=-\sin \theta d s_{1}$, la seconde par $d \eta=\cos \theta d s_{1}$, et ajoutons, il vient :

$$
d \pi-2 \mathrm{C} d \theta=0, \text { le long } \operatorname{des}\left(\mathrm{I}_{\mathrm{I}}\right)
$$

De même, multiplions la première équation (6) par :

$$
d \xi=-\cos \theta d s_{2}
$$

la seconde par :

$$
d \eta=-\sin \theta d s_{2}
$$

et ajoutons, il vient :

$$
d \pi+2 \operatorname{Cd} \theta=0 \quad \text { le long } \operatorname{des}\left(\Gamma_{2}\right)
$$

En résumé, aux lignes de glissement $\left(\Gamma_{1}\right)$ et $\left(\Gamma_{2}\right)$ de première et de seconde espèce, sont respectivement attachés les systèmes différentiels :

$$
\begin{aligned}
& \left\{\begin{array}{l}
\cos \theta d \xi+\sin \theta d \eta=0 \\
d \pi-2 \mathrm{C} d \theta=0
\end{array}\right. \\
& \left\{\begin{array}{l}
\sin \theta d \xi-\cos \theta d \eta_{i}=0 \\
d \pi+2 \mathrm{C} d \theta=0
\end{array}\right.
\end{aligned}
$$

Si $(\gamma)$ n'est pas ligne de glissement du massif, par tout point $M$ intérieur au quadrilatère formé par quatre lignes de glissement, deux à deux issues des extrémités de $\gamma$ (fig. 22), passent deux lignes de glissement rencontrant $\gamma$ en $\alpha$ et $\beta$ entre ses extrémités A et B. Chacune de ces lignes établit une relation entre les valeurs de $\pi$ et $\theta$ en $M$;

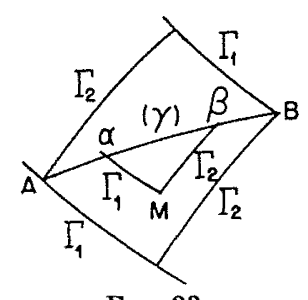

FiG. 22

$\pi$ et $\theta y$ sont donc bien déterminées. Au contraire, pour un point extérieur au quadrilatère des lignes de glissement, on ne peut mener qu'une seule ou zéro ligne de glissement rencontrant $(\gamma)$. Suivant

(*) Ces relations traduisent en fait l'équilibre du quadrilatère infinitésimal de côtés $d s_{1}$ et $d s_{2}$, formé par l'intersection de deux lignes $\left(\Gamma_{1}\right)$ et de deux lignes $\left(\Gamma_{2}\right)$ infiniment voisines. les régions du plan, la solution est soil déterminée, soit simplement ou doublement indéterminée.

Remarque. - Les lignes de glissement apparaissent ainsi, du point de vue analytique, comme les lignes caractéristiques du système d'équation aux dérivées partielles (6). Le raisonnement fail montre qu'elles sont réelles. Les relations (7) et (8), qui expriment les conditions pour que le problème de CaUchr, généralement impossible à partir de données arbitraires de $\pi$ et $\theta$ le long d'une ligne caractéristique, soit indéterminé, sont dites caractéristiques du système en cause.

\section{Propriétés générales des lignes de glisse-} MENT ET DE CERTAINES FONCTIONS S'Y RATTACHANT.

L'existence d'un réseau de lignes de glissement ayant été mis en évidence, il semble d'autant plus indiqué d'y rapporter le massif en vue de l'étude de son état limite qu'il constitue un syslème naturel de coordonnées orthogonales particulièrement approprié à cette fin.

Soient donc, relativement au repère primilif $x$ o $y$ par rapport auquel les coordonnées d'un point quelconque d'une ligne de glissement sont toujours désignées par $\xi \eta$ :

$$
u(\xi n)=\mathrm{C}^{12}, \quad v(\xi n)=\mathrm{C}^{\prime \prime}
$$

les équations des lignes de glissement de seconde espèce $\left(\Gamma_{2}\right)$ et de première espèce $\left(\Gamma_{1}\right)$ respective-

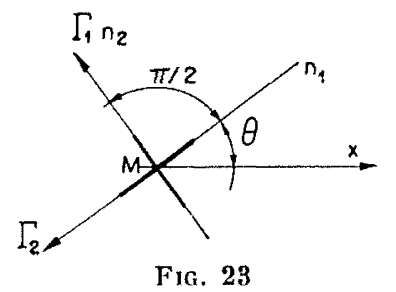

ment. Ces dernières sont orientées comme précisé sur la figure 23, et par l'introduction des quantités positives $\mathrm{E}_{1}, \mathrm{E}_{2}$,

définies par :

$\mathrm{E}_{1}{ }^{2}=\left(\frac{\partial \xi}{\partial u}\right)^{2}+\left(\frac{\partial r_{1}}{\partial u}\right)^{2}, \mathrm{E}_{2}{ }^{2}=\left(\frac{\partial \xi}{\partial v}\right)^{2}+\left(\frac{\partial r_{1}}{\partial v}\right)^{2}$,

leurs arcs élémentaires onl pour expressions :

$$
d s_{1}=\mathrm{E}_{1} d u\left(\operatorname{pour} \Gamma_{1}\right), \quad d s_{9}=\mathrm{E}_{2} d v\left(\text { pour } \Gamma_{2}\right)
$$

La mise en œuvre des définitions nouvelles ainsi précisées conduit très directement à quelques propriétés importantes qui vont être exposées, 
a) Transformation des équations caractéristiques.

Aux systèmes (7) et (8), relatifs aux lignes de glissement, se substituent ainsi les équations :

$$
\begin{aligned}
& \left\{\begin{array}{l}
\frac{\partial \xi}{\partial u}+E_{1} \sin \theta=0, \frac{\partial \eta_{1}}{\partial u}-E_{1} \cos \theta=0 \\
\frac{\partial \pi}{\partial u}-2 C \frac{\partial \theta}{\partial u}=0, \quad \text { (le long de toute } \Gamma_{1} \text { ) }
\end{array}\right. \\
& \left\{\begin{array}{l}
\frac{\partial \xi}{\partial v}+E_{2} \cos \theta=0, \frac{\partial \eta}{\partial v}+E_{2} \sin \theta=0 \\
\frac{\partial \pi}{\partial v}+2 \mathrm{C} \frac{\partial \theta}{\partial v}=0, \quad \text { (le long de toute } \Gamma_{2} \text { ) }
\end{array}\right.
\end{aligned}
$$

\section{b) Courbure des lignes de glissement.}

La courbure $k_{1}$ des lignes $\left(\Gamma_{1}\right)$ et celle $k^{2}$ des lignes $\left(\Gamma_{2}\right)$, qui ont respectivement pour valeurs :

$$
k_{1}=\frac{1}{\mathrm{E}_{1}} \frac{\partial \theta}{\partial u}, \quad k_{2}=\frac{1}{\mathrm{E}_{2}} \frac{\partial \theta}{\partial v},
$$

satisfont à des équations différentielles intégrables qui vont être formées. A cet effet, l'identification des dérivées secondes $\partial^{2} \xi / \partial u \partial v$, déduites des premières équations (12) et (13) donne :

$$
\begin{aligned}
-\sin \theta \frac{\partial \mathrm{E}_{1}}{\partial v}-\mathrm{E}_{1} \cos \theta \frac{\partial \theta}{\partial v}=- & \cos \theta \frac{\partial \mathrm{E}_{2}}{\partial u} \\
& +\mathrm{E}_{2} \sin \theta \frac{\partial \theta}{\partial u}
\end{aligned}
$$

et de même par la considération des dérivées $\partial^{2} \eta / \partial u \partial v$, il vient : :

$\cos \theta \frac{\partial \mathbf{E}_{1}}{\partial v}-\mathbf{E}_{1} \sin \theta \frac{\partial \theta}{\partial v}$

$$
=-\sin \theta \frac{\partial E_{2}}{\partial u}-E_{2} \cos \theta \frac{\partial \theta}{\partial u}
$$

soit en résolvant en $\frac{\partial \mathrm{E}_{1}}{\partial v}$ et $\frac{\partial \mathrm{E}_{2}}{\partial u}$ :

$$
\frac{\partial \mathbf{E}_{1}}{\partial v}=-\mathbf{E}_{2} \frac{\partial \theta}{\partial u}, \quad \frac{\partial \mathbf{E}_{2}}{\partial u}=\mathbf{E}_{1} \frac{\partial \theta}{\partial v}
$$

puis, compte tenu de (14) :

$$
k_{1}=-\frac{1}{\mathrm{E}_{1} \mathrm{E}_{2}} \frac{\partial \mathrm{E}_{1}}{\partial v}, \quad k_{2}=\frac{1}{\mathrm{E}_{1} \mathrm{E}_{2}} \frac{\partial \mathrm{E}_{2}}{\partial u}(16)
$$

Dérivant ensuite chacune des équations (15) par $\partial u$ et $\partial v$ respectivement, il vient pour valeur commune de :

$$
\frac{\partial^{2} \theta}{\partial u \partial v}=k_{1} \frac{\partial \mathrm{E}_{1}}{\partial v}+\mathrm{E}_{1} \frac{\partial k_{1}}{\partial v}=k_{2} \frac{\partial \mathrm{E}_{2}}{\partial u}+\mathrm{E}_{2} \frac{\partial k_{2}}{\partial u},
$$

Or, les relations caractéristiques (deuxièmes équations (12) et (13)) dérivées, soit :

$$
\frac{\partial^{2} \pi}{\partial u \partial v}-2 \mathrm{C} \frac{\partial^{2} 0}{\partial u \partial v}=0, \frac{\partial^{2} \pi}{\partial u \partial v}+2 \mathrm{C} \frac{\partial^{2} \theta}{\partial u \partial v}=0
$$

entrainent la nullité de $\left(\partial^{2} \theta / \partial u \partial v\right)=0$, ce qui, par (17), assigne aux courbures de satisfaire au système :

$$
\frac{1}{\mathrm{E}_{2}} \frac{\partial k_{1}}{\partial v}-k_{1}^{2}=0, \frac{1}{\mathrm{E}_{1}} \frac{\partial k_{2}}{\partial u}+k_{2}^{2}=0
$$

qui, puisque :

$$
\frac{1}{\mathrm{E}_{2}} \frac{\partial k_{1}}{\partial v}=\frac{d k_{1}}{d s_{2}}, \frac{1}{\mathrm{E}_{1}} \frac{\partial k_{2}}{\partial u}=\frac{d k_{2}}{d s_{1}},
$$

devient finalement :

$$
\frac{\partial k_{1}}{d s_{2}}-k_{1}^{2}=0, \frac{\partial k_{2}}{d s_{1}}+k_{2}^{2}=0 .
$$

Sous cette forme, l'intégration annoncée est immédiate et, en désignant par $\rho_{1}$ et $\rho_{2}$ les rayons de courbure des lignes de glissement $\left(\Gamma_{1}\right)$ et $\left(\Gamma_{2}\right)$, donne les relations :

$$
e_{1}+s_{2}=\mathrm{C}^{\text {te }}, \quad \rho_{2}-s_{1}=\mathrm{C}^{\mathrm{te}},
$$

propres à permettre le tracé des lignes de glissement à partir, par exemple, de deux lignes de glissement de chaque système se coupant en un point.

\section{c) Recherche directe des fonctions $\pi$ et $\theta$.}

Le problème de la recherche des lignes de glissement et par suite celui de la détermination des fonctions $\pi$ et $\theta$, à partir des données de celle-ci sur une courbe du massif [3] sont ainsi géométriquement résolus. Ce dernier peut toutefois être abordé d'une autre manière, et les conséquences qui s'ensuivent présentent quelque intérêt.

Considérons les relations caractéristiques (12) et (13). En toute généralité, $f(v)$ et $g(u)$ désignant deux fonctions arbitraires des variables, elles s'indiquent par :

$$
\pi-2 \mathrm{C} \theta=f(v), \quad \pi+2 \mathrm{C} \theta=g(u)
$$

Plusieurs cas doivent être envisagés :

$1^{\circ} f$ et $g$ dépendent effectivement des variables.

Par un changement de fonctions convenables, il est alors possible de réduire le système précédent à :

$$
\pi-2 \mathrm{C} 0=\mathrm{C} v, \quad \pi+2 \mathrm{C} \theta=\mathrm{C} u,
$$

d'où :

$$
\pi=\mathrm{c} \frac{u+v}{2}, \quad 0=\frac{u-v}{2}
$$


Ainsi, la connaissance des coordonnées curvilignes $u$ et $v$ d'un point du massif équivaut à celle des fonctions $\pi$ et $\theta$ en ce point. Or, dans le réseau coordonné $\left(\Gamma_{1}, \Gamma_{2}\right)$, celles-ci sont lićes aux abscisses curvilignes $s_{1}, s_{2}$ par les équations (11) intégrées, si bien qu'en définitive toute la question est régie par les fonctions $\mathrm{E}_{1}(u \cdot v) \quad \mathrm{E}_{2}(u . v)$. C'est l'équation aux dérivées partielles commune, à laquelle chacune d'elles satisfait, qui va maintenant être formée. Compte tenu de (21), la dérivation déjà effectuée des premières équations (12) et (13) relativement à $u$ et $v$ donne :

$$
\begin{aligned}
\frac{\partial^{2} \xi}{\partial u \partial v}=\frac{1}{2} \mathrm{E}_{1} \cos \theta & -\sin \theta \frac{\partial \mathrm{E}_{1}}{\partial v} \\
& =\frac{1}{2} \mathrm{E}_{2} \sin \theta-\cos \theta \frac{\partial \mathrm{E}_{2}}{\partial u}
\end{aligned}
$$$$
\frac{\partial^{2} \eta}{\partial u \partial v}=\frac{1}{2} \mathrm{E}_{1} \sin \theta+\cos \theta \frac{\partial \mathrm{E}_{1}}{\partial v}
$$$$
=-\frac{1}{2} \mathrm{E}_{2} \cos \theta-\sin \theta \frac{\partial \mathrm{E}_{2}}{\partial u}
$$

d'où :

$$
\mathrm{E}_{2}=-2 \frac{\partial \mathrm{E}_{1}}{\partial v}, \quad \mathrm{E}_{1}=-2 \frac{\partial \mathrm{E}_{2}}{\partial u}
$$

soit par séparation des fonctions, les deux équations du second ordre cherchées :

$$
\frac{\partial^{2} \mathrm{E}_{1}}{\partial u \partial v}-\frac{1}{4} \mathrm{E}_{1}=0, \frac{\partial^{2} \mathrm{E}_{n}}{\partial u \partial v}-\frac{1}{4} \mathrm{E}_{2}=0
$$

identiques, du type classique des télégraphistes, et pour l'intégration desquelles on se reportera aux traités d'analyses.

$2^{\circ} f$ et $g$ se réduisent à des constantes $a$ et $b$.

$\pi$ et $\theta$ sont alors constants dans tout le milieu, les lignes de glissement constituent un réseau de droites orthogonales. C'est le cas de Rankine évoqué ci-dessus. Il convient de noter que par la définition de $\pi=\mathfrak{c}+\Phi \Phi+\delta y$, cette éventualité implique des conditions aux limites convenables tant pour les pressions $(\mathscr{V} \mathcal{C})$ que pour l'écoulement de filtration ( $\Phi)$ qui règne dans le massif.

$3^{\circ}$ Une seule des deux fonctions, par exemple $g$, se réduit à une constante $b$. Les deux relations caractéristiques qui s'intègrent par :

$$
\pi-2 \mathrm{C} \theta=f(v), \quad \pi+2 \mathrm{C} \theta=b
$$

donnent :

$$
2 \pi=f(v)+b, \quad 2 \mathrm{C} \theta=b-f(v)
$$

q étant indépendant de $u$, l'intégration de la première équation (7) donne, par la fonction arbitraire $h(l)$ :

$$
\cos \theta \dot{5}+\sin \theta \eta=h(v)
$$

Les lignes de première espèce $\left(\Gamma_{1}\right)$ constituent un faisceau de droites à un paramètre, tangentes à une courbe (C) (fig. 24). Les lignes de se-

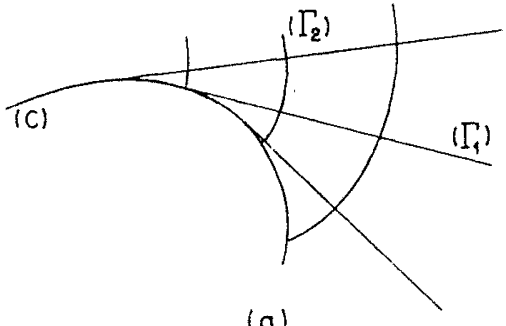

(a)

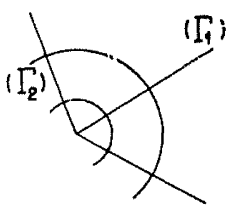

(b)
FiG. 24

conde espèce $\left(\Gamma_{2}\right)$ trajectoires orthogonales des $\left(\Gamma_{1}\right)$ sont les développantes de $(\mathrm{C})$. Le long d'une ligne de première espèce, $\pi$ est constant, ce qui implique comme en $2^{\circ}$ des conditions de chargement et un potentiel d'écoulement particuliers. Cette circonstance présente un cas de dégénérescence lorsque $(\mathrm{C})$ s'évanouit ponctuellement. Les $\left(\Gamma_{1}\right)$ sont alors les rayons des cercles concentriques $\left(\Gamma_{2}\right)$ (fig. $24 b$ ).

\section{Equilibre limite parallète.}

Les généralités théoriques développées ci-dessus sont précisées sur le cas particulier de l'équilibre limite parallèle pour lequel, par définition, les fonctions $g r=1 / 2\left(N_{1}+N_{2}\right)$ et 0 demeurent constantes le long de droites parallèles à l'axe $\mathrm{OX}$ faisant l'angle $i$ avec l'horizontale $o x$ (fig. 25).

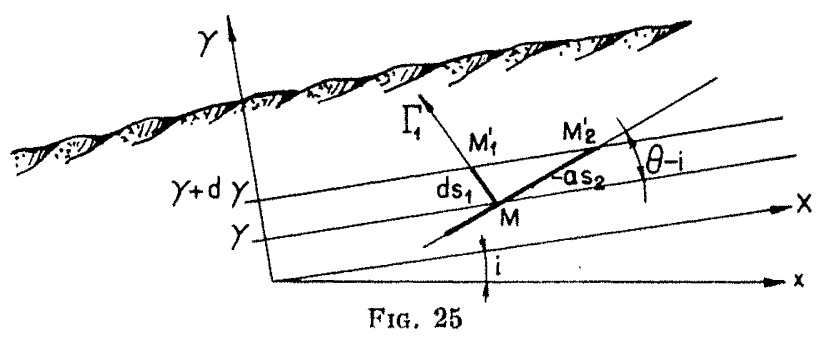

Cette situation est notamment à considérer pour la détermination des dimensions limites des talus susceptibles d'en assurer la stabilité. Soient M un point du massif d'ordonnée $\gamma$ et les lignes de glissement de ce point $\left(\Gamma_{1}\right)$ et $\left(\Gamma_{2}\right)$ qui coupent la droite d'ordonnée $\gamma+d \gamma$ respectivement en $\mathrm{M}_{1}^{\prime}$ et $\mathrm{M}_{2}^{\prime}$. Le long de chacun des éléments de ces lignes, de longueur $d s_{1}$ et $d s_{2}$, les ordonnées $\xi$ et $\eta$ de $M$ subissent des variations :

$$
\begin{cases}d \xi_{1}=-\sin \theta d s_{1}, & d \eta_{1}=\cos \theta d s_{1} \\ d \xi_{2}=-\cos \theta d s_{2} . & d \eta_{2}=-\sin \theta d s_{2}\end{cases}
$$


et la variation d'ordonnée a manifestement pour expressions :

$$
d \gamma=-\sin (\theta-i) d s_{2}=\cos (\theta-i) d s_{1}
$$

D'autre part, les variations de $\mathscr{T}$ el de 0 sont les mêmes dans les mêmes conditions, et leurs valeurs, relatives à la droite d'ordonnée $\gamma+d \gamma$, satisfont aux équations simultanées :

$$
\left\{\begin{array}{l}
d \mathfrak{x}-2 \mathrm{C} d 0+d \mathrm{\Upsilon}_{1}=0 \\
d \mathfrak{x}+2 \mathrm{C} d \theta+d \mathrm{\Upsilon}_{2}=0
\end{array}\right.
$$

conséquences des relations caractéristiques et où $d \Upsilon_{1}$ et $d \Upsilon_{2}$ désignent respectivement les variations de $\Upsilon=\bar{\omega} \Phi+\delta y$ le long de $\left(\Gamma_{1}\right)$ et $\left(\Gamma_{2}\right)$ à partir du point $M$. Ces dernières quantités, compte tenu de (25), ont d'ailleurs pour valeurs :

$$
\left\{\begin{array}{l}
d \Upsilon_{1}=-\frac{\partial \Upsilon^{r}}{\partial \xi} \sin \theta d s_{1}+\frac{\partial \Upsilon^{r}}{\partial \eta} \cos \theta d s_{1}, \\
d \Upsilon_{2}=-\frac{\partial \Upsilon^{\circ}}{\partial \xi} \cos \theta d s_{2}-\frac{\partial \Upsilon^{r}}{\partial \eta} \sin \theta d s_{2},
\end{array}\right.
$$

soit encore par (26) :

$$
\begin{aligned}
& d \Upsilon_{1}{ }_{1}=\left(-\frac{\partial \Gamma}{\partial \xi} \sin \theta+\frac{\partial \Gamma}{\partial \eta} \cos \theta\right) \frac{d \gamma}{\cos (\theta-i)} \\
& =\frac{\partial \Upsilon^{\circ}}{d s_{1}} \frac{d \gamma}{\cos (0-i)} \\
& d r_{2}=\left(\frac{\partial Y^{\circ}}{\partial \xi} \cos \theta+\frac{\partial Y}{\partial \eta} \sin \theta\right) \frac{d \gamma}{\sin (0-i)} \\
& =\frac{\partial \Upsilon^{\top}}{d s_{2}} \frac{d \gamma}{\sin (\theta-i)}
\end{aligned}
$$

Explicitant les dérivées partielles de $x^{*}$ par l'introduction de la vitesse de filtration :

$$
\overline{\mathrm{V}}=-\mathrm{K} \overline{\operatorname{grad}} \Phi,
$$

(K coefficient de perméabilité du sol homogène et isotrope) d'intensité $\mathrm{V}$, faisant angle $\chi$ avec $o x$ en posant :

$$
\frac{\bar{\omega} \mathrm{V}}{\mathrm{K} \delta}=\lambda
$$

il vient :

$$
\frac{\partial \Upsilon}{\partial \xi}=-\delta \lambda \cos \alpha, \frac{\partial \Upsilon}{\partial \eta}=-\delta \lambda \sin \alpha+\delta,
$$

puis :

$$
\left\{\begin{array}{l}
d \Upsilon_{1}=\delta[\lambda \sin (\theta-\alpha)+\cos \theta] \quad \frac{d \gamma}{\cos (\theta-i)} \\
d \Upsilon_{2}=\delta[-\lambda \cos (\theta-\alpha)+\sin 0] \frac{d \gamma}{\sin (\theta-i)}
\end{array}\right.
$$

Enfin par (31), le système (27) devient :

$\left\{\begin{array}{l}d \mathfrak{x}-2 \mathrm{C} d \theta+\delta[\lambda \sin (\theta-\alpha)+\cos \theta] \frac{d \gamma}{\cos (\theta-i)}=0 \\ d \mathfrak{T}+2 \mathrm{Cd} \theta+\delta[-\lambda \cos (\theta-\alpha)+\sin \theta] \frac{d \gamma}{\sin (\theta-i)}=0\end{array}\right.$

Sa résolution en $d \gamma$ et $d \mathfrak{r}$ donne :

$$
\begin{aligned}
d \gamma & =\frac{2 \mathrm{C}}{\mu \cdot \hat{o}} \sin 2(0-i) d \theta, \\
d \mathscr{T} & =\frac{2 \mathrm{C}}{\mu}[\sin (2 \theta-i)-\lambda \cos (2 \theta-x-i)] d 0
\end{aligned}
$$

avec :

$$
\mu=\lambda \cos (i-\alpha)-\sin i .
$$

L'intégration nécessite la connaissance des fonctions $\lambda$ et $\mu$, e'est-à-dire celle du champ des vitesse de filtration. Supposons, ce qui est toujours licite à titre d'approximation, celui-ci uniforme dans la zone plus ou moins étendue du massif considéré. Dans ces conditions, $\lambda$ et $\mu$, comme $\mathrm{V}$ et $\alpha$, sont constantes et les équations (33) et (34) intégrées deviennent :

$$
\begin{aligned}
\gamma & =-\frac{\mathrm{C}}{\mu \cdot \delta} \cos 2(0-i)+\mathrm{C}^{\mathrm{te}}, \\
x & =-\frac{\mathrm{C}}{\mu}[\cos (20-i)+\lambda \sin (20-\alpha-i)]+\mathrm{C}^{\mathrm{ie}} .
\end{aligned}
$$

Ces dernières précisent complètement l'état d'équilibre envisagé par ses lignes de glissement et l'intensité des contraintes en tout point. Les lignes de glissement sont d'ailleurs susceptibles d'une définition géométrique précise. En effet, rapportant celles-ci aux axes OX, OY sur chaque $\left(\Gamma_{1}\right), d \mathrm{X}$ et $d \mathrm{Y}$ sont liés par :

$$
d \mathrm{X}=-\operatorname{tg}(\theta-i) d \mathrm{Y}
$$

d'où, par (33) :

$$
d \mathrm{X}=-\frac{\mathrm{C}}{\mu . \delta} \sin ^{2}(0-i) d 0
$$

et par intégration :

$$
\mathrm{X}=-\frac{\mathrm{C}}{y . \delta}\left[\frac{\theta-i}{2}-\frac{1}{2} \sin 2(\theta-i)\right]+\mathrm{C}^{\mathrm{te}}
$$

Posant :

$$
2(\theta-i)=\omega,
$$

et compte tenu de (36), s'obtiennent ainsi les 
équations paramétriques des lignes de glissement de première espece $\left(\Gamma_{1}\right)$ :

$$
\left\{\begin{array}{l}
\mathrm{X}=-\frac{\mathrm{C}}{\mu \cdot \delta}(\omega-\sin \omega)+\mathrm{C}^{\mathrm{te}} \\
\mathrm{Y}=-\frac{\mathrm{C}}{\mu \delta} \cos \omega+\mathrm{C}^{\mathrm{te}} .
\end{array}\right.
$$

Par un calcul analogue, et puisque sur les lignes de glissement de seconde espéce $\left(\Gamma_{2}\right) d \mathrm{X}$ et $d \mathrm{Y}$ sont liés par :

$$
d \mathrm{X}=\operatorname{cotg}(\theta-i) d \mathrm{Y}
$$

il vient pour celles-ci :

$$
\left\{\begin{array}{l}
\mathrm{X}=\frac{\mathrm{C}}{\mu \delta}(\omega+\sin \omega)+\mathrm{C}^{\mathrm{te}}, \\
\mathrm{Y}=-\frac{\mathrm{C}}{\mu \delta} \cos \omega+\mathrm{C}^{\mathrm{te}} .
\end{array}\right.
$$

En (39) et (40) se reconnaît la représentation d'un réseau évidemment quadratique, constitué de cycloïdes égales engendrées par des cercles roulant sans glisser sur des parallèles à $\mathrm{OX}$ et de rayons égaux $\mathrm{C} / \mu \delta$.

Cette propriété est source de conséquences pratiques importantes sur lesquelles nous aurons à insister au chapitre suivant consacré aux applications de la théorie ici exposée.

\section{III}

\section{APPLICATION DE LA THÉORIE DES SOLS COHÉRENTS SANS FROTTEMENT INTERNE}

1.

Les applications de la théorie exposée au precédent chapitre sont, à vrai dire, fort nombreuses sinon illimitées, et toute tentative d'en dresser un catalogue complet s'avérerait fastidieuse et illusoire.

C'est donc à l'étude détaillée de quelques exemples typiques que se limilent les développements actuels, désignant par le terme de vase les sols de l'espèce envisagée, pour lesquels l'angle de frottement interne est nul ou négligeable et la cohésion appréciable; nous examinerons successivement, et pour les massifs bidimensionnels :

- Ie poinçonnement d'une couche indéfinie de vase par une construclion fondée en quelque région de sa surface;
- L'action d'un massif de vase sur un rideau rigide;

-.. Les conditions d'équilibre des talus et la limitation de leurs dimensions compatibles avec leur stabilité.

A cet effet, seront prises en considération, en sus des sollicitations superficielles, des forces de volume d'origine gravifiques et hydrauliques et dérivant du potentiel :

$$
\Upsilon=\bar{\omega}+\delta y
$$

défini en [2.II.1].

2. Charge de poinçonnement D'Une Covche de VASE DE PROFONDEUR INDÉFINIE.

Le massif de rase de cohésion $\mathrm{C}$, limité à sa partic supérieure par l'horizontale $O x$, est indé-

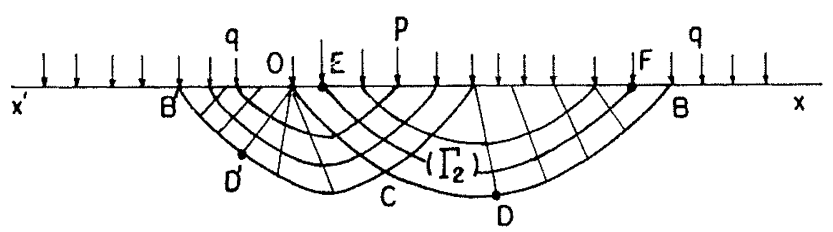

FIG. 26

fini dans la direction de cet axe et vers le bas (fig. 26).

Il esl sollicité par :

Des forces de volume de potentiel $\gamma^{\prime}(1)$, en provenance de la pesanteur et d'un ćcoulement de filtration de charges $\Phi$;

- Des forces superficielles comportant un chargement uniforme d'inlensité q par unité de longueur des demi-droites $O x^{\prime}$ et $\Delta x$.

Il s'agit de déterminer à quelle condition un cquilibre limite $d u$ massif peut intéresser une zone finie de celui-ci, autrement dit de préciser la relation existant entre les intensités $p$ et $q$ lorsqu'une telle circonstance est réalisée. Dans cette éventualité, une ligne de glissement partant de $\mathrm{O}$ aboutit en un point $\mathrm{B}$ de $\mathrm{A} x$ pour délimiter avec $O x$ la zone $O C B$ en équilibre limite. De même une ligne de glissement part de $A$ pour aboutir en $B^{\prime}$ sur $O x^{\prime}$ délimitant avec $O x$ la zone $\mathrm{ACB}^{\prime}$ en équilibre limite. L'allure des lignes de glissement données par la figure 26 et correspondant à l'intuition la plus directe met en lumière plusieurs domaines présentant des caractères propres : (OCA) est en état de poussée sous la pression verticale la plus forte $p,(\mathrm{OCD})$ et $\left(\mathrm{OCI}^{\prime}\right)$ sont en état de butée, sous la pression verticale la plus faible $q,(\mathrm{OCD})$ ct $\left(\mathrm{OCD}^{\prime}\right)$ sont des zones de passage d'un type d'état à l'autre dans les- 
quelles les lignes d'une famille rayonnent de chacun des points singuliers $O$ et $A$.

$\mathrm{Au}$-dessous de la ligne $\mathrm{B}^{\prime} \mathrm{CB}$, le massif est en équilibre surabondant.

Portons notre attention sur une ligue de glissement située au-dessus de OCD, partant de $\mathrm{E}$ sous le chargement $p$ et aboutissant en $\mathrm{F}$ sous le chargement $q$. C'est une $\left(\Gamma_{2}\right)$ comme il résulte de l'état des contraintes en $\mathrm{E}$ et $\mathrm{F}$ faisant l'objet des figures $27 a$ et $27 b$ respectivement, d'où se

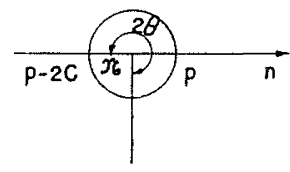

(a)

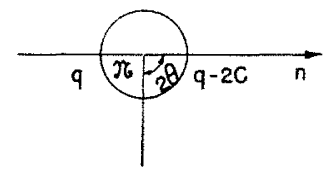

(b)
FIG. 27

déduisent encore les valeurs des fonctions $\mathscr{T}$ et $\theta$ en chacun de ses deux points, soit :

$$
\begin{aligned}
& \text { En } \mathrm{E}: \mathfrak{x}=p-c, \quad \theta=\frac{3 \pi}{4} \\
& \text { En } \mathrm{F}: \mathfrak{x}=q+c, \quad 0=\frac{\pi}{4}
\end{aligned}
$$

Le long de cette ligne de glissement de seconde espèce, est invariable la quantité [2.II.3] :

$$
\pi+2 \mathrm{C} \theta=\mathscr{x}+\bar{\omega} \Phi+\delta y+2 \mathrm{C} \theta,
$$

d'où, par prise en considération de chacune de ses extrémités où le potentiel $\Phi$ a les valeurs connues $\Phi_{0}$ en $\mathrm{E}, \Phi_{1}$ en $\mathrm{F}$ :

$$
p=q+\mathrm{C}(2+\pi)-\bar{\omega}\left(\Phi_{0}-\Phi_{1}\right)
$$

Applicatron. - Soit un massif rigide fondé à la profondeur $h$ au-dessous de la surface horizontale d'un sol de l'espèce et formant barrage entre deux bassins communiquant par le substratum perméable et dont les plans d'eau présentent la différence de niveau $e$ (fig. 28).

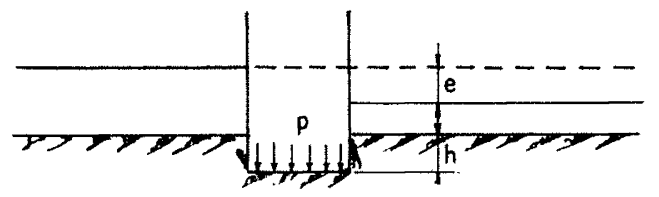

Fig. 28

La valeur de la charge $p$ admissible au niveau de la fondation du barrage résulte de la formule (2) dans laquelle :

$$
q=\delta h, \quad \Phi_{0}-\Phi_{1} \# e
$$

d'où :

$$
p=\delta h+\mathrm{C}(2+\pi)-\bar{\omega} e
$$

Cas particulier. - En l'absence de courant de filtration, la condition d'équilibre se résume ¿videmment à :

$$
p=q+\mathrm{C}(2+\pi)
$$

Nous laissons au lecteur le soin d'établir comme conséquence de [2.II.4] que dans cette circonstance, par ailleurs bien classique, le réseau des lignes de glissement symétrique par rapport à la médiatrice de $\mathrm{OA}$ se compose de segments de droites et d'arcs de cercles, tels ceux représentés sur la figure 29 avec correspondance à la figure 22 des zones désignées par les mêmes lettres.

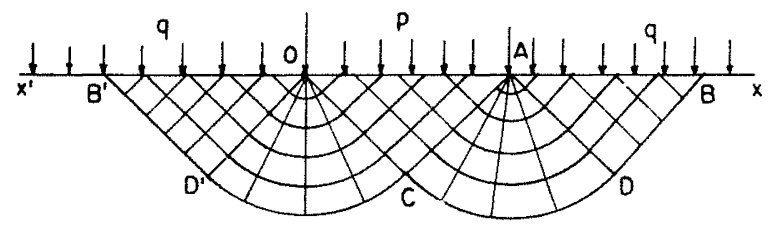

Fig. 29

3. Action D'une COUGhe dF vase chargée sur UN RIDEAU RIGIDE.

Il s'agit d'un masif de vase de cohésion $\mathrm{C}$, limité à sa partie supérieure par l'horizontale $\mathrm{O} x$ chargée verticalement par les forces verticales d'intensité uniforme $p$, s'appuyant sur un réseau rigide $\mathrm{OB}$ de fruit $i$. Diverses circonstances sont à envisager, qui donnent lieu, en ce qui concerne la distribution des pressions $q$ le long de OB, correspondant à l'état limite du massif, aux résultats ci-dessous.

\section{a) Rideau parfaitement lisse.}

La contrainte sur tout élément du massif en contact avec le rideau se réduit à sa composante normale $q$. Deux cas sont à distinguer suivant la nature de l'équilibre limite régnant dans la zone $\mathrm{OAB}$ limitée vers le bas par la ligne de glissement issue du pied du rideau.

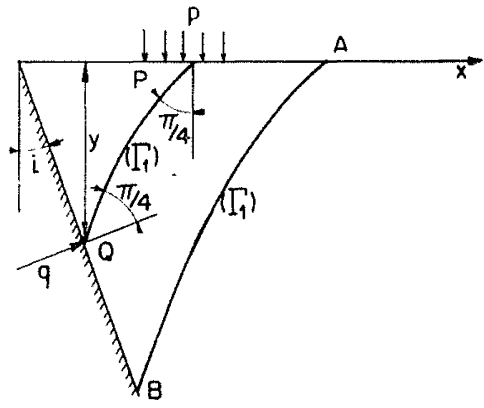

Fxa. 30

$1^{\circ}$ Poussée (fig. 30-31 $a-31 b$ ) :

Toute ligne de glissement $P Q$ issue d'un 
point $Q$ du rideau et aboutissant sur $\mathrm{O} x$ est de première espèce et les valeurs de $\mathscr{r}$ et de $\theta$ en chacune de ses extrémités sont:

En P : $\mathscr{x}=p-\mathrm{C}, \quad \theta=\frac{3 \pi}{4}$

En $Q: \mathfrak{r}=q+\mathrm{C}, \quad \theta=i+\frac{3 \pi}{4}$

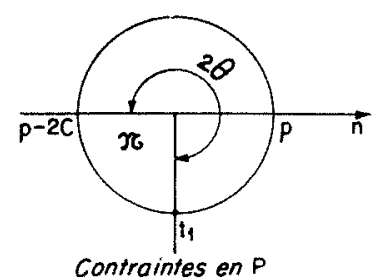

(a)

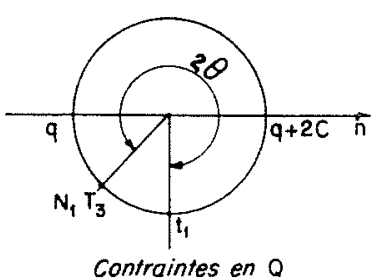

(b)
Fig, 31

Par [2.II.3], l'expression :

$$
\pi-2 \mathrm{C} \theta=\mathscr{C}+\Upsilon-2 \mathrm{C} \theta
$$

a même valeur en $P$ et en $Q$. Désignant par $\Upsilon_{0}$ et $X_{1}$ la valeur de $Y=\bar{\omega} \Phi+\delta y$ en chacun de ces points, on en déduit la pression normale :

$$
q=p-2 \mathrm{C}(1-i)+\mathrm{\Upsilon}_{0}-\mathrm{\Upsilon}_{1},
$$

dont la connaissance suppose, par le terme en $\Phi$, le mode d'écoulement de filtration régnant éventuellement dans le massif :

Si l'eau du massif est au repos, à une constante près, $\Upsilon$ se réduit au potentiel $\delta y$ des forces gravifiques et en tout point $Q$ du rideau situé à la profondeur $y$ au-dessous de $\mathrm{O} x$, la pression est :

$$
q=p+\delta y-2 \mathrm{C}(1-i)
$$

$2^{\circ}$ Butée (fig. 32-33a-33 b) :

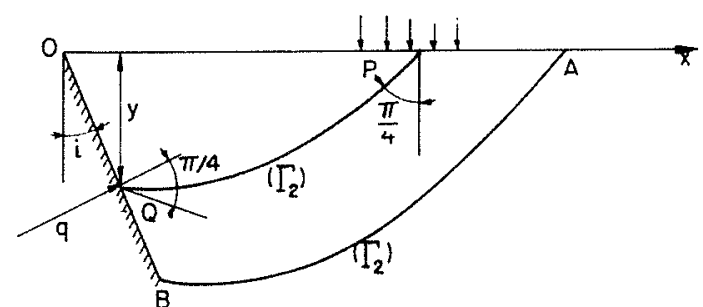

Fig. 32

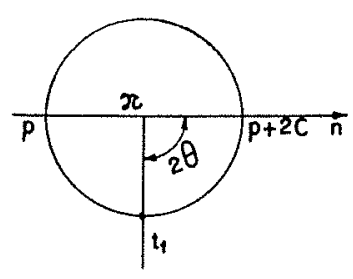

Contraintes en $\mathrm{P}$

(a)

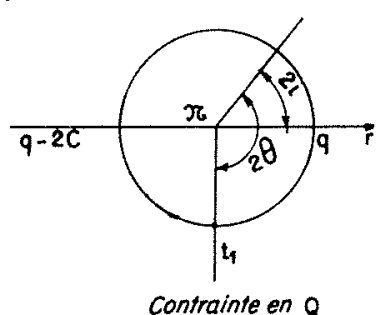

(b)
Fic. 33
La ligne de glissement $P Q$ est de seconde espèce et, en $\mathrm{P}$ :

$$
\mathfrak{r}=p+\mathrm{C}, \quad \theta=\pi / 4
$$

en $\mathbf{Q}$ :

$$
x=q-c, \quad 0=\pi / 4+i .
$$

La constance de $\pi+2 \mathrm{C} \theta$ donne comme cidessus :

$$
q=p+2 \mathrm{C}(1-i)+\mathrm{r}_{0}-\mathrm{r}_{1}
$$

soit, en l'absence d'écoulement et pour tout point du rideau de profondeur $y$ :

$$
q=p+\delta y+2 \mathrm{C}(1-i)
$$

\section{b) Rideau parfaitement rugueux.}

La composante tangentielle $t$ de la contrainte s'exerçant sur tout élément du massif en contact avec le rideau prend la valeur maximum compatible avec la stabilité de la vase, c'est-à-dire celle de la cohésion $\mathrm{C}$.

$1{ }^{\circ}$ Poussée (fig. 34-35 $a-35 b$ ) :

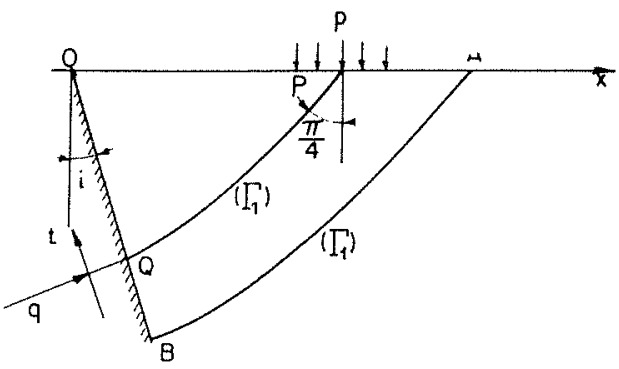

FIG. 34

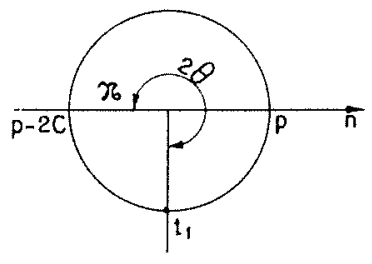

Contraintes en $\mathrm{P}$

(a)

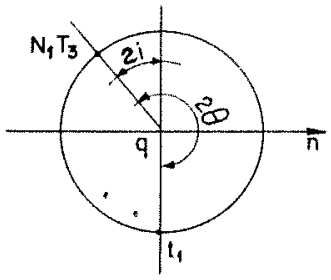

Contraintes en 0

(b)
Fig. 35

La composante $t$ est dirigée de $B$ vers $O$. Toute ligne de glissement $P Q$ est de première espéce, et comme :

$$
\text { en } \mathrm{P}: \mathfrak{r}=p-\mathrm{C}, \quad 0=\frac{3 \pi}{4},
$$




$$
\text { En } Q: \mathfrak{T}=q, \quad 0=\frac{\pi}{2}+i,
$$

La constance de $\pi-2 \mathrm{C} \theta$ donne la valeur de la composante normale $q$ de lia constante en $Q$ :

$$
q=p-2 \mathrm{C}\left(\frac{2+\pi}{4}-i\right)+\mathrm{x}_{0}-\mathrm{x}_{1}
$$

soit en l'absence de courant :

$$
q=p+\circ y-2 \mathrm{C}\left(\frac{2+\pi}{4}-i\right)
$$

$2^{\circ}$ Butée (fig. $36-37 a-37 b$ ) :

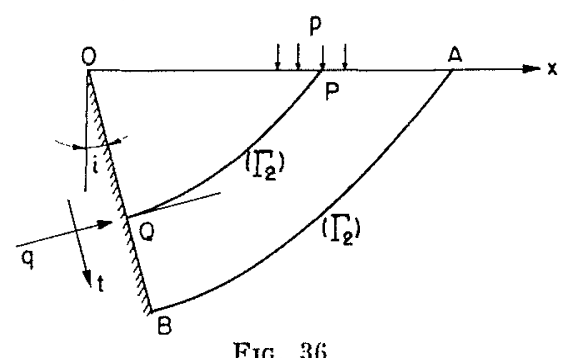

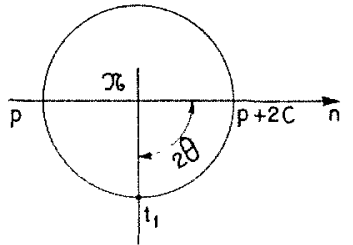

Contraintes en $P$

(a)

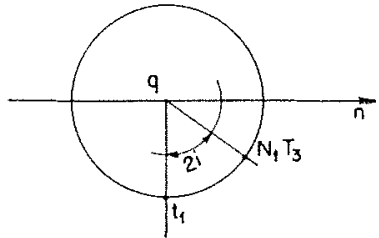

Contraintes en 0

(b)
Fig. 37

La composante $t$ est dirigée de $O$ vers $B$. Toule ligne de glissement $\mathrm{PQ}$ est de seconde espèce.

$$
\begin{array}{ll}
\text { En } \mathrm{P}: \mathfrak{x}=p+\mathrm{C}, & \theta=\frac{\pi}{4}, \\
\text { En } \mathrm{Q}: \mathfrak{r}=q, & 0=i,
\end{array}
$$

d'où :

$$
q=p+2 \mathrm{C}\left(\frac{2-\pi}{4}-i\right)+\mathrm{r}_{0}-\mathrm{r}_{1}
$$

soil en l'absence de courant:

$$
q=p+\delta y+2 \mathrm{C}\left(\frac{2-\pi}{4}-i\right),
$$

c) Rideau mobilisant partiellement le frottement.

Sur tout élément du massif en contact avec le rideau, les composantes normales $q$ et tangentielles $t$ de la contrainte satisfont à une relation de la forme $t=q \operatorname{tg}$ : où frottement mobilisé.
1"Poussée (fig. 38 et 39) :

Toute ligne de glissement PQ est de première espèce. Les valeurs de $\mathscr{r}$ el de $\theta$ en $\mathrm{P}$ sont les mèmes que pour le cas $b, 1^{\circ}$ ), soil :

$$
x=p-\mathrm{C}, \quad 0=\frac{3 \pi}{4}
$$

En $Q$, les contraintes donnent lieu à la représentation plane, objet de la figure 39, d'où :
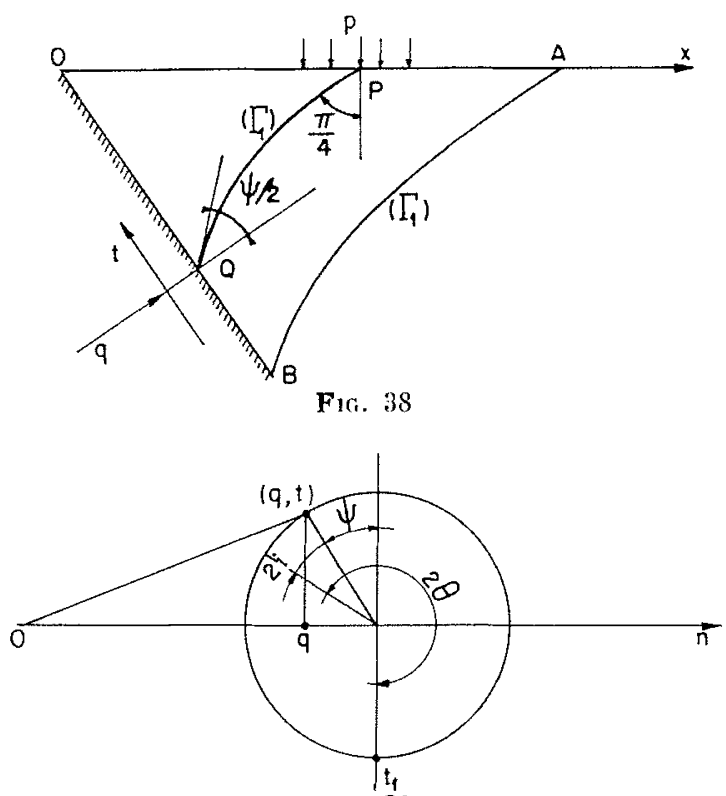

FIG. 39

$$
\Re=q+\mathrm{C} \sin \psi, \quad 0=i+\frac{\pi}{2}+\psi
$$

avec :

$$
\cos \psi=\frac{t}{\mathrm{C}}=\frac{q \lg \rho}{\mathrm{C}}
$$

Par la constance de $\pi-2 \mathrm{C} 0$, on déduit de ces résultats :

$$
q=p-\mathrm{C}\left(\frac{\pi}{2}-\psi-2 i+\sin \psi\right)+\mathrm{r}_{0}-\mathrm{\Upsilon}_{1}
$$

soit, si le massif n'est le siège d'aucun écoulement :

$$
q=p+d y-\mathrm{C}\left(\frac{\pi}{2}-\psi-2 i+\sin \psi\right)
$$

\section{2" Butée :}

Le cas de la butée, par des développements analogues, donne lieu aux résultats :

$$
\begin{gathered}
q=p+\mathrm{C}\left(\frac{\pi}{2}-\psi-2 i+\sin \psi\right)+\Upsilon_{0}-\Upsilon_{\mathrm{y}} \\
q=p+\delta y+\mathrm{C}\left(-\frac{\pi}{2}-\psi-2 i+\sin \psi\right)
\end{gathered}
$$


Remarque. - Aux éventualités $(a)(b)(c)$ examinées ci-dessus correspondent respectivement trois dispositions différentes de soutènement d'un massif de vase : par un rideau de palplanches (a), par un massif en enrochements ou en maçonnerie très rugueuse $(b)$, par un massif de sable de frottement interne.

\section{STAbilité des Talus.}

Toujours comme application très directe de la théorie générale exposée au chapitre précédent, nous nous proposons d'examiner diverses circonstances ayant trait à l'équilibre des talus et dont certaines dispositions naturelles, ou intervenant lors de certains aménagements, présentent de nombreux exemples.

Seront ainsi envisagés les problèmes suivants :

- Détermination des conditions d'équilibre d'un talus éventuellement noyé et siège d'un écoulement de filtration;

- Détermination de la profondeur limite d'un approfondissement par dragage;

Détermination de la forme d'un talus d'égale résistance au risque de glissement.

Rappelons brièvement les résultats essentiels à mettre en œuvre à cet effet.

Le massif de poids spécifique $\Delta$, de module des vides $m$, comprenant de l'eau de poids spécifique () dont la charge en tout point est $\Phi=(p / \sigma)+y$ (Oy verticale ascendante) est sollicité par des forces de volume dérivant du potentiel $\Upsilon=\bar{\omega} \Phi=-\delta \mathrm{y}$ avec $\hat{o}=\Delta-\bar{\omega}(1-m)$, et par des forces de surface ou charges appliquées sur sa surface limite, exception faite des pressions hydrostatiques éventuelles complées dans grad $r$. Si la phase solide existe seule, $r$ se réduit à $\Delta y$. Si la phase liquide est au repos, la charge $\Phi$ est partout constante, et le potentiel $\Upsilon$, à une constante près, se réduit à $\delta y$. Désignnant par $\mathscr{r}$ la pression moyenne en tout point et posant $\pi=\mathfrak{r}+\Upsilon$, le long d'une ligne de glissement $\left(\Gamma_{1}\right)$ faisant avec $O x$ (horizontale) l'angle $0+(\pi / 2)$ et le long d'une ligne de glissement $\left(\Gamma_{2}\right)$ faisant l'angle $0+\pi$ avec cet axe, sont respectivement constantes les quantités $\pi-2 \mathrm{C} 0 \mathrm{ct}$ $\pi+2 \mathrm{C} 0$. Tout ceci suppose une courbe intrinsèque du sol d'équation $t= \pm \mathrm{C}$, c'est-à-dire celui-ci assez comprimé pour ne pas courir le risque de rupture par traction. Cette condition n'est pas nécessairement vérifiée en tout point, et il convient d'y prendre garde. Au voisinage de la surface libre notamment, il sera utile de considérer une tranche de massif fissurée verticalement $\left(^{\star}\right)$, d'épaisseur $a=\left(2 \mathrm{C}_{i} / \delta\right)$, telles qu'à son

(*) Il est possible de montrer, par un raisonnement direct calqué sur [2.II.3] et tenant compte de la forme de la courbe intrinsèque au voisinage de l'origine, que niveau inférieur où viennent mourir les lignes de glissement de la zone sous-jacente (fig. 40), l'étal des contraintes (fig. 41) soit compatible avec un ćtat comprimé de celle-ci.

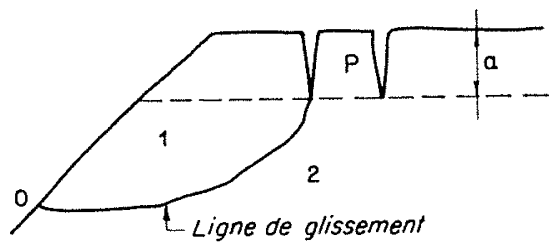

Fig. 40

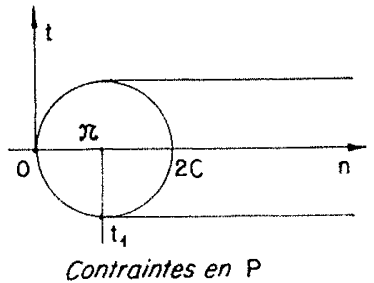

Fig. 41

Ceci étant, la méthode mise en ouvre dans les exemples concrets qui suivent est loujours la même, et consiste à écrire que l'expression $\pi-2 \mathrm{CO}(\pi+2 \mathrm{C} 0)$ prend la même valeur aux deux extrémités, où est connu l'état des contraintes, d'une ligne de glissement $\Gamma_{1}\left(\Gamma_{2}\right)$ frontière commune tracée intuitivement dans le massif du domaine en équilibre limite (1) et de la zone en équilibre surabondant (2).

a) Equilbre d'un talus (fig. $42-43 a-43 b$ ) :

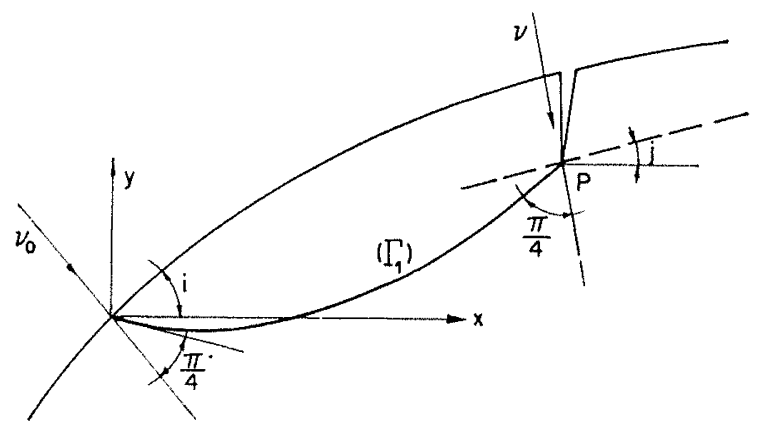

FIG. 42

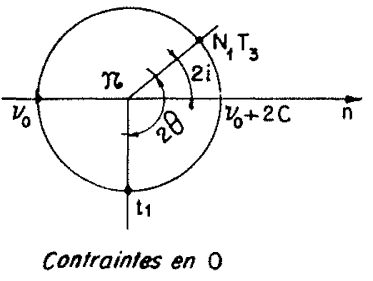

(a)

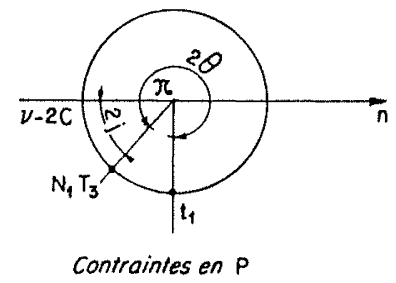

(b)
Fig. 43

Soient O le pied du talus de pente $i$ en O, OP la ligne de glissement limile, $j$ la pente du talus

les fissures sont effectivement verticales au voisinage de la surface libre, quelle que soit l'inclinaison de celle-ci. Ce fait est conforme à l'observation courante. 
en P, $N_{0}$ et $N$ les pressions supposées normales au talus (exclusion faite de la pression hydrostatique éventuelle) en $\mathrm{O}$ et en $\mathrm{P}$. La ligne de glissement OP est de première espèce. D'autre part :

$$
\begin{array}{ll}
\text { En } O: \mathfrak{C}=N_{0}+\mathrm{C}, & 0=\frac{\pi}{4}+i, \\
\text { En P : } \mathscr{x}=N-\mathrm{C}, & 0=\frac{3 \pi}{4}+i,
\end{array}
$$

D'où par la constance de $\pi-2 \mathrm{C} \theta$ et en notant $\Upsilon_{0}$ et $\Upsilon_{1}$ la valeur de $\Upsilon$ en $O$ et $P$ respectivement :

$$
\begin{aligned}
\pi-2 \mathrm{C} \theta=N_{0} & +\mathrm{C}+\mathrm{\Upsilon}_{0}-2 \mathrm{C}\left(\frac{\pi}{4}+i\right) \\
& =N-\mathrm{C}+\Upsilon-2 \mathrm{C}\left(\frac{3 \pi}{4}+j\right)
\end{aligned}
$$

soit :

$$
2 \mathrm{C}(i-j)=\mathrm{C}(2+\pi)+N_{0}+\Upsilon_{0}-\left(N_{1}+\Upsilon_{1}\right)
$$

Dans le sens de la sécurité, les lignes de glissement les plus défavorables sont celles pour lesquelles $\mathrm{P}$ est situé sous le terre-plein horizontal. Dans ces conditions, $j=0$, et la condition d'équilibre devient :

$$
\mathrm{C}(2+\pi-2 i)=N_{1}+\Upsilon_{1}-\left(N_{0}+\Upsilon_{0}\right)
$$

$N_{1}$ y comporte, en plus des charges éventuelles appliquées au terre-plein, la charge $\delta_{a}\left(\Delta_{a}\right)$ de la partie fissurée située au-dessus de $P$.

La pression $N_{0}$ correspond par exemple à un chargement du pied de talus destiné à améliorer la stabilité.

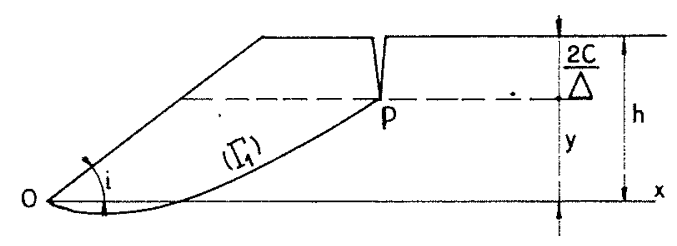

FIG. 44

Exemple (fig. 44). - Soit un talus sec non chargé pour lequel $\Upsilon=\Delta y$. L'équation (9), où :

$$
N_{0}=0 \quad N_{1}=2 \mathrm{C}, \quad \mathrm{r}_{0}=0, \quad \mathrm{r}_{1}=\Delta y_{1}
$$

donne pour la hauteur dangereuse totale du talus :

$$
h=y_{1}+2 \frac{\mathrm{C}}{\Delta}=\frac{\mathrm{C}}{\Delta}(2+\pi-2 i) .
$$

\section{b) Approfondissement par dragage :}

L'approfondissement par dragage d'un bassin à fond vaseux ne peut se poursuivre au-delà d'une certaine limite correspondant à l'annulation de la pente $i$ du pied de talus. L'équation (9) détermine cette grandeur dont voici les expressions correspondant aux dispositions les plus courantes.

$1^{\circ}$ Massif complètement noyé en équilibre hydrostatique (fig. 45).

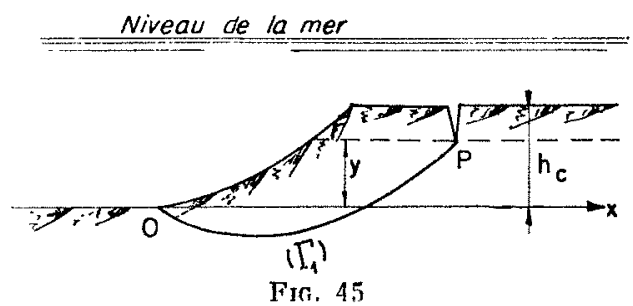

En $O: \Upsilon_{0}=0, \quad N_{0}=0$;

En P : $\Upsilon_{1}=\delta y, \quad N_{1}=2 \mathrm{C}$

D'où par (9), dans laquelle $i=0$ :

$$
\mathrm{C}(2+\pi)=2 \mathrm{C}+\delta y,
$$

soit pour la profondeur limite totale :

$$
h_{l}=y+\frac{2 \mathrm{C}}{\delta}=\frac{\mathrm{C}(2+\pi)}{\Delta-\bar{\omega}(1-m)}
$$

$2^{\circ}$ Massif à terre-plein émergeant (fig. 46).

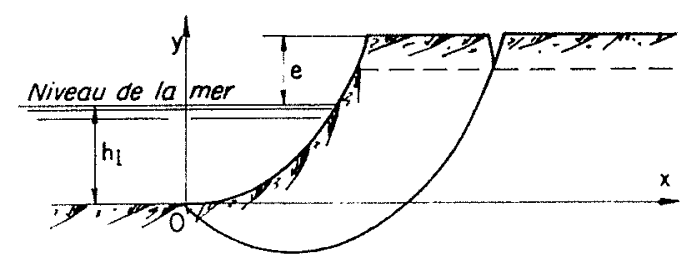

FIG. 46

Soit $h_{l}$ la cote de la surface des eaux, comptée à partir du fond du bassin, $e$ l'émergence du terre-plein. Toujỏurs dans l'hypothèse d'un équilibre hydrostatique entre l'eau du bassin et celle du massif, on peut assigner au potentiel $\Upsilon$ les valeurs :

$\Upsilon=\delta y$, pour $\mathrm{O} \leqslant y \leqslant h$, et $\Upsilon=\delta h_{l}+\Delta\left(y-h_{l}\right)$, pour $h \leqslant y \leqslant e-(2 \mathrm{C} / \Delta)$. Ainsi en $\mathrm{O}: \Upsilon_{0}=0$, $N_{0}=0$, et en $\mathrm{P}: \Upsilon_{1}=\delta h_{l}+\Delta\left(y_{1}-h_{l}\right)$, $N_{1}=2 \mathrm{C}$. Comme $y_{1}=h_{1}+e-(2 \mathrm{C} / \Delta)$, il vient par (9) où $i=0$ :

$$
h_{l}=\frac{\mathrm{C}(2+\pi)-\Delta e}{\Delta-\bar{\omega}(1-m)}
$$


c) Stabilité au voisinage d'une résurgence.

Nous envisageons un massif baigné par une nappe d'eau de niveau (L), siège d'un écoulement de surface libre $(\Lambda)$, sensiblement horizontale à quelque distance du talus $\mathrm{OA}$ avec laquelle elle se raccorde tangentiellement au point de résurgence $R$, situé au-dessus de (L). La ligne de glissement de première espèce $O P$ coupe $(\Lambda)$ au point $Q$ de cote $h_{Q}$ à la profondeur $e$ au-dessous du terre-plein, pour aboutir en $P$ à la base de la tranche fissurée d'épaisseur $a$ (fig. 47). Nous nous

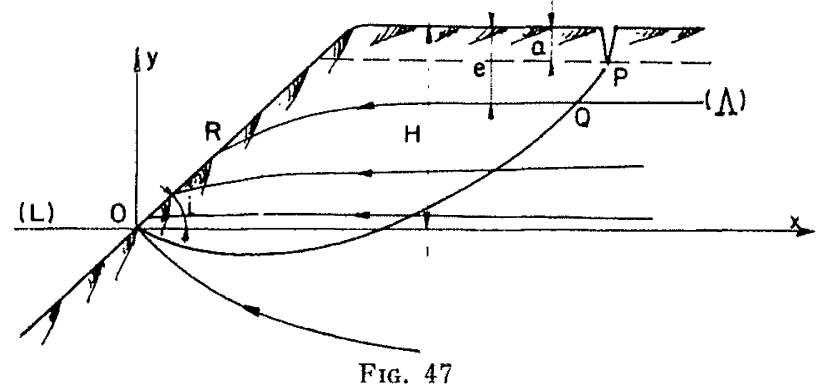

proposons de calculer la hauteur $\mathrm{H}$ du massif au-dessus du plan d'eau (L), pour laquelle l'équilibre limite précédant l'éboulement de la zone OAP est atteint dans celle-ci. A cet effet, nous faisons état de la relation (9).

Pour :

$$
0 \leqslant y \leqslant h_{0}, \quad x=\varpi \Phi+\delta y,
$$

et pour :

$$
h_{\mathrm{Q}} \leqslant y \leqslant h_{\mathrm{Q}}+e, \quad \Upsilon^{2}=\Upsilon_{\mathrm{Q}}+\Delta\left(y-h_{\mathrm{Q}}\right) .
$$

D'où :

$$
\text { En } \mathrm{O}: \Upsilon_{0}=0, \quad N_{0}=0
$$

En P:

$$
\mathrm{J}_{\mathrm{r}}=(\varpi+\delta) h_{\mathrm{Q}}+\Delta\left(h_{1}, \cdots-h_{0}\right), \quad N_{1}=\Delta u,
$$

et par (9) :

$\mathrm{G}(2+\pi-2 i)=(\Delta+m \pi) h_{12}+\Delta\left(h_{p}-h_{Q_{2}}+a\right)$

soit, puisque :

$$
\begin{gathered}
\mathrm{H}=h_{\mathrm{P}}+a=h_{\mathrm{Q}}+e \\
\mathrm{H}=\frac{\mathrm{C}(2+\pi-2 i)+m \omega e}{\Delta+m \sigma}
\end{gathered}
$$

d) Forme d'un talus d'égale résistance au risque de glissement.

Dans les divers cas envisagés ci-dessus, on peut se proposer de déterminer une forme de talus $O A$, telle qu'en tout point de celui-ci l'inclinaison $i$ satisfasse précisément à la relation d'équilibre (9) où $C$ est frappé d'un coefficient de sécurité $k$. L'origine des axes étant au point du talus situé à la base de la tranche fissurée d'épais seur $a=(2 \mathrm{C} / \delta)$, il vient ainsi :

$$
k \mathrm{C}(2+\pi-2 i)=(\Upsilon+N)^{\circ} y
$$

A titre d'exemple, bornons-nous au cas $\left(b, 1^{\circ}\right)$ de l'approfondissement par dragage d'une fosse bordée par un massif complètement noyé (fig.48).

En $\mathrm{O}: \mathrm{\gamma}_{0}=0, \quad N_{0}=\delta a$.

En tout point $M(x, y)$ :

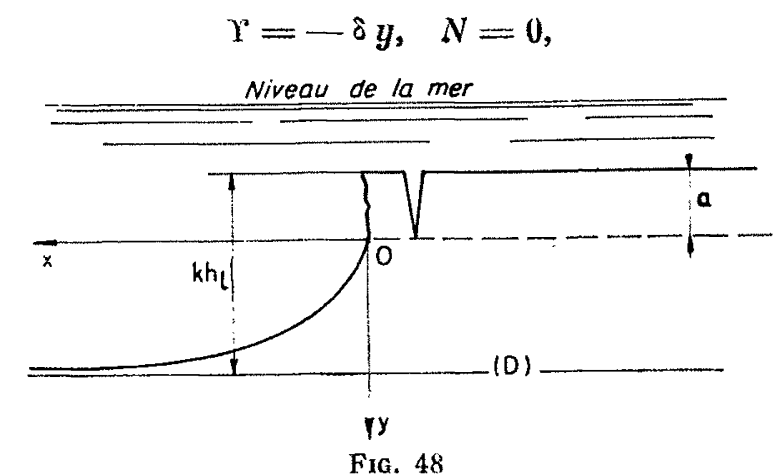

d'où par (13) :

$$
i=1+\frac{\pi}{2}-\frac{2 \delta}{k \mathrm{C}}(y+a)
$$

Soit en posant $\lambda=(k \mathrm{C} / 2 \delta)$ et par intégration l'équation cartésienne du profil OM du talus :

$$
x=-\lambda \frac{\log \cos \left(1-\frac{y+a}{\lambda}\right)}{\log \cos \left(1-\frac{a}{\lambda}\right)}
$$

Nous ne nous étendrons pas sur le tracé de ce dernier et nous nous contenterons de remarquer qu'il admet comme asymptote horizontale la droite (D) située à la profondeur $k h l(10)$ audessous du terre-plein.

\section{DIMENSIONS limites DES MASSifs.}

Soit un massif de profil provisoirement indéterminé dans le demi-plan situé d'un même côté de la droite (5), de pente $i$, qui lui sert de frontière. Il est sollicité, en plus des forces de volume dérivant du potentiel $Y$ et comprenant celui d'un champ de filtration uniforme de vitesse $\bar{V}$ (nodule $\vec{V}$, orientation $\alpha$ ) et celui des actions gravifiques, par un chargement superficiel normal à $\mathrm{S}$, d'intensité $\mathrm{P}_{0}$. Nous supposons qu'une région nécessairement finie du massif, limitée par une portion de la droite (S) et par divers ares de lignes de glissement $(\Gamma)$, est en équilibre limite parallele [2.II.5] (fig. 49). La frontière de cette 
zone est manifestement celle à assigner au profil du massif pour que celui-ci soit à sa limite de rupture par glissement, sous l'hypothèse énoncée quant à la nature de l'équilibre précédant ce phé-

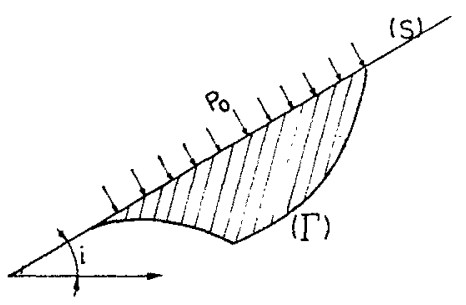

Fig. 49

nomène. Nous nous proposons d'en déterminer les dimensions (longueur $l$, profondeur $h$ ). Rappelons à cet effet que nous avons posé $\lambda=(\bar{\omega} \mathrm{V}) /(\mathrm{K} \delta)$ (2.II.30) et $u=\lambda \cos (i-\alpha)-\sin i(2 . I I .35)$ pour désigner les paramètres propres à définir le champ de filtration uniforme régnant dans le massif. Nous avons reconnu que les lignes de glissement de tout équilibre limite parallèle constituent un réseau quadratique de cycloïdes ígales. Si bien que la fronticre $(\Gamma)$ de la zone cherchée est formée d'ares de telles courbes représentées par les équations paramétriques (*) (2.II.39 40):

$$
\begin{aligned}
& \left(\Gamma_{1}\right)\left\{\begin{array}{l}
\mathrm{X}=-\frac{\mathrm{C}}{\mathrm{y} \cdot \delta}(\omega-\sin \omega)+\mathrm{C}^{+\omega} \\
\mathrm{Y}=-\frac{\mathrm{C}}{\mathrm{y} \cdot \delta} \cos (1)+\mathrm{C}^{\mathrm{te}}
\end{array}\right. \\
& \left(\Gamma_{2}\right)\left\{\begin{array}{l}
\mathrm{X}=\frac{\mathrm{C}}{\omega \cdot \delta}(\omega+\sin (\omega) \\
\mathrm{Y}=-\frac{\mathrm{C}}{1 \cdot \delta} \cos \omega+\mathrm{C}^{\mathrm{o}}
\end{array}\right.
\end{aligned}
$$

Pour la délerminer, deux cas sont à distinguer suivant que le massif est amené à se rompre par butée ou par poussée.

\section{1'Butée.}

Le chargement $P_{0}$ a la valeur minimum. Dans

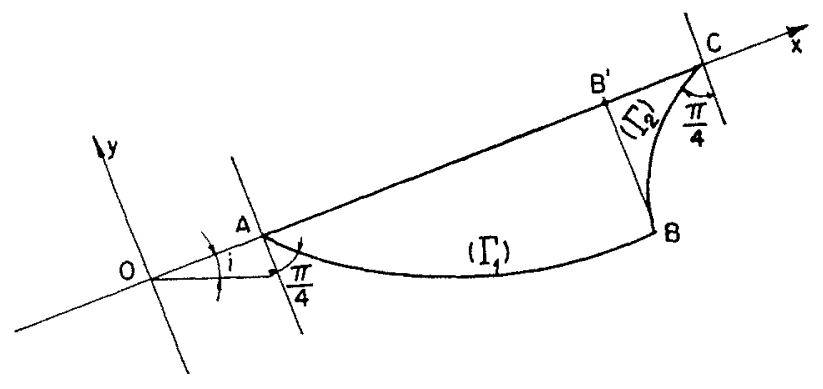

F1(i. 50

(*) Où l'on a posé : $\omega=2(\theta-i)$ (2.II.38). ces conditions, $(\Gamma)$ comporte : un arc de première espèce $\left(\Gamma_{1}\right)$ issu du point $A$ de $S$ et aboutissant en B sommet de la cycloïde qui le contient; un arc de seconde espèce $\left(\Gamma_{2}\right)$ issu en $B$ et aboutissant au point $C$ de $S$ (fig. 50). En tout point de (S) et notamment en $\mathrm{A}$ et $\mathrm{C}$ :

$$
\begin{gathered}
\theta=(\pi / 4)-i, \omega=(\pi / 2) . \\
\operatorname{En} B, \theta=(\pi / 2)+i, \omega=\pi .
\end{gathered}
$$

Dis lors, et par les équations paramétriques rappelées ci-dessus, il vient :

$$
\mathrm{AB}^{\prime}=-\frac{\mathrm{C}}{\mu \delta}\left(\frac{\pi}{2}+1\right), \mathrm{B}^{\prime} \mathrm{C}=-\frac{\mathrm{C}}{\mu \delta}\left(\frac{\pi}{2}-1\right),
$$

d'où :

$$
l=\mathrm{AC}=\cdots \frac{\mathrm{C} \pi}{\mu . \delta}
$$

et,

$$
h=\mathrm{BB}^{\prime}=-\frac{\mathrm{C}}{\mu \hat{o}}
$$

\section{2" Poussée.}

Le chargement $P_{0}$ a la valeur maximum. Dans ces conditions, $(\Gamma)$ comporte deux arcs de cyclö̈des $A B$ et $B C$ tels ceux représentés ci-dessous

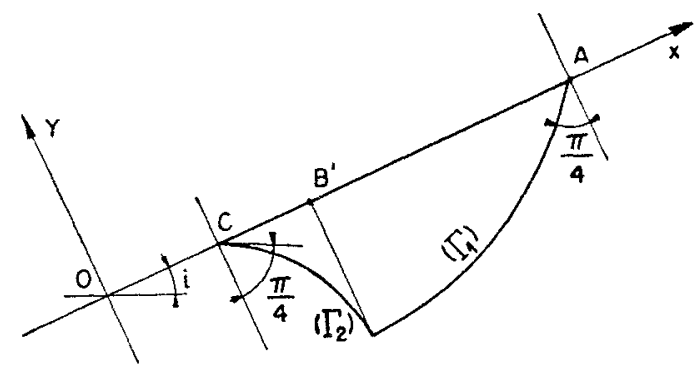

Fir. 51

(fig. 51). Le long de (S), et notamment en $\Lambda$ et $\mathrm{C}$ :

$$
0=-(\pi / 4)+i, \quad \omega=-(\pi / 2) .
$$

En $B: 0=-(\pi / 2)+i, \quad \omega=-(\pi / 2)$.

D’où, par les équations paramétriques des $\left(\Gamma_{1}\right)$ et $\left(\Gamma_{2}\right)$ :

$$
\begin{array}{r}
\mathrm{AB}^{\prime}=\frac{\mathrm{C}}{\mu \delta}\left(1+\frac{\pi}{2}\right), \quad \mathrm{B}^{\prime} \mathrm{C}=\frac{\mathrm{C}}{\mu \delta}\left(\frac{\pi}{2} \cdots 1\right), \\
\mathrm{AC}=\frac{\mathrm{C} \pi}{\mu \delta}, \quad \mathrm{BB}^{\prime}=\frac{\mathrm{C}}{\mu \delta}
\end{array}
$$

Les dimensions limites cherchées sont ainsi les mêmes dans les deux cas envisagés. Ce sont cel- 
les qui ne sauraient être dépassées sous peine d'entraîner la rupture par glissement, en quelque région affleurant à sa surface, du massif de l'espèce. Leur dépendance au paramètre u. relatif au champ de filtration permet d'apprécier, par exemple, le risque de glissement que peut présenter un talus initialement stable par suite de précipitations qui y provoquent un écoulement de vitesse estimable.

Exemple D'application. - Soit un massif limité par un talus $\mathrm{AA}^{\prime}$ d'inclinaison $i$ comportant une zone perméable d'épaisseur $e$, de direction générale parallèle à $\mathrm{AA}^{\prime}$ formée d'un sol de poids spécifique réduit $\delta$, de cohésion $C$. Il s'agit d'estimer les risques de glissement d'ensemble qu'il présente à l'action d'un courant liquide de poids spécifique $\bar{\omega}$ déterminée dans la zone perméable par une alimentation à l'infini amont (région A) et une résurgence à l'infini aval (région $A^{\prime}$ ) (fig. 52).

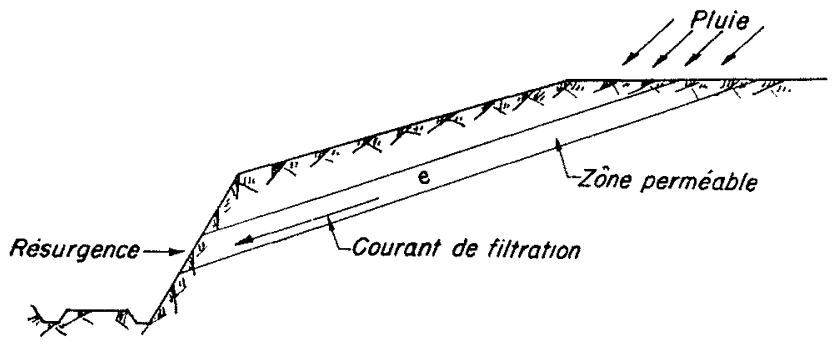

FIG. 52

Supposons l'équilibre limite atteint dans la zone perméable. Il est nécessairement parallèle et de poussée à quelque distance des singularités d'extrémités (alimentation et résurgences).

Dans ces conditions :

$$
\operatorname{grad} \Phi=-\sin i,
$$

$\operatorname{par}\left(I_{30}\right):$

$$
\lambda=\frac{\bar{\omega}}{\delta} \sin i
$$

par $\left(\mathrm{I}_{35}\right)$ :

$$
\mu=-\left(1+\frac{\bar{\sigma}}{\delta} \sin i\right),
$$

L'épaisseur $e$ de la zone perméable, suffisante pour y permettre l'établissement de l'état d'équilibre limite, et par suite de déterminer la ruine de l'ouvrage en cause par glissement d'ensemble, résulte de (20), soit :

$$
e=\frac{\mathrm{C}}{(\sigma+\delta) \sin i}
$$

\section{IV}

\section{EQUILIBRES LIMITES DES SOLS PULVÉRULENTS FILTRANTS}

1. L'étude des sols pulvérulents (sables), dont l'angle de frottement interne $\varphi$ est fini et la cohésion nulle, a donné lieu à des développements classiques relativement étendus et d'autant plus intéressants qu'ils concernent aussi les sols pulvérulents et cohérents (argiles) puisque les résultats relatifs aux premiers s'étendent très directement aux seconds par la mise en œuvre du théorème de Caquot, dit des états correspondants $\left(^{*}\right)$. Mais les équations différentielles régissant les états limites de tels milieux, à l'encontre de ce qui a été constaté pour les sols dépourvus de frottement interne, ne sont généralement pas intégrables; aussi serons-nous contraints, dans le cadre de cet exposé écrit en vue des applications, de nous borner à l'exposé de quelques-unes de leurs propriétés particulières de portée pratique étendue. A cette fin, nous supposons comme à l'ordinaire tout massif de l'espèce soumis à des actions superficielles connues, et à des forces de volume dérivant du potentiel $\mathrm{Y}=\bar{\Phi} \Phi+\delta y$ (oy verticale ascendante) dont les composantes :

$$
\begin{aligned}
& \mathbf{X}=-\frac{\partial \Upsilon}{\partial x}=-\bar{\omega} \frac{\partial \Phi}{\partial x}, \\
& \mathbf{Y}=-\frac{\partial \Upsilon}{\partial y}=-\bar{\omega} \frac{\partial \Phi}{\partial y}-\delta,
\end{aligned}
$$

seront désignées par :

$$
\mathrm{X}=\mathrm{A} \sin \alpha, \quad \mathrm{Y}=-\mathrm{A} \cos \alpha
$$

de telle sorte que leur intensité ait pour mesure $\mathrm{A}$ et que leur azimuth relatif à la direction de la verticale descendante soit $\alpha$ (fig. 53). En

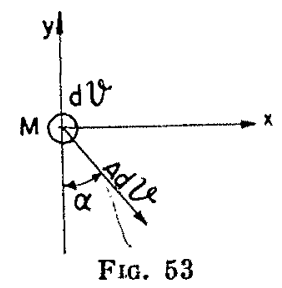

toute généralité, A et $\alpha$ dćpendent de la particule $(M, d v)$ considérée. Comme grad $\Phi$, ces quantités sont des constantes lorsqu'est uniforme le champ de filtration dont le massif est siège.

(*) Le cas singulier pour lequel ce théorème n'est pas applicable est précisément celui des vases, et fait l'objet du chapitre II du présent article. 


\section{EQvations indéfINIES D'ÉQUILIBRE LIMITE.}

Rappelons qu'est défini comme limite tout état pour lequel, en chaque point du massif, l'inclinaison maximum de la contrainte sur la normale à l'élément qui lui correspond est égale à l'angle de frottement interne $\bullet$ de la terre qui le constitue. Le cercle représentatif des contraintes s'y trouve ainsi, par définition, tangent aux droites de Covlomb : $t= \pm n \operatorname{tg} \varphi$, les deux points de contact $t_{1}$ et $t_{2}$ étant images des élément sur lesquels l'inclinaison de la contrainte est $\varphi$ (fig. 54). Ces éléments, dits de glissement,

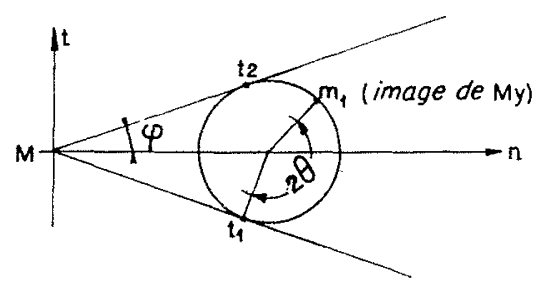

FIG. 54

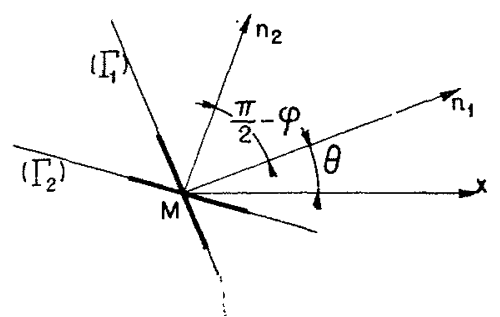

FIG. 55

font entre eux l'angle $(\pi / 2)-\varphi$ (fig. 55). Les lignes de glissement qui leur sont tangentes constituent ainsi un réseau réel de deux familles distinctes $\left(\Gamma_{1}\right)$ et $\left(\Gamma_{2}\right)$, d'ares $s_{1}$ et $s_{2}$. Par tout point du massif passent deux telles courbes; leur angle est $(\pi / 2)-\varphi$. Soit 0 l'angle de la direction $\left(r_{1}\right)$ avec la verticale ascendante, $p$ la valeur de la pression, mesurée par $\mathrm{M} t_{1}=\mathrm{M} t_{2}$, s'exerçant sur chaque élément de glissement. Des considérations géométriques simples, déduites de l'examen de la figure 55, en posant $\bar{\omega}=2 \theta-\varphi$, donnent les relations :

$$
\begin{aligned}
& \mathbf{N}_{1}=\frac{p}{\cos \varphi}(1+\sin \varphi \sin \omega), \\
& \mathrm{N}_{2}=\frac{p}{\cos \varphi}(1-\sin \varphi \sin \omega), \\
& \mathrm{T}_{3}=-p \operatorname{tg} \varphi \cos \omega .
\end{aligned}
$$

qui définissent l'état d'équilibre limite en fonction des deux paramètres $p$ et 0 . Par l'introduction de ceux-ci, les équations indéfinies de l'équilibre:

$$
\begin{aligned}
& \frac{\partial \mathrm{N}_{1}}{\partial x}+\frac{\partial \mathrm{T}_{3}}{\partial y}+\frac{\partial \Upsilon}{\partial x}=0 \\
& \frac{\partial \mathrm{T}_{3}}{\partial x}+\frac{\partial \mathrm{N}_{2}}{\partial y}+\frac{\partial \Upsilon}{\partial y}=0
\end{aligned}
$$

s'écrivent :

$$
\left\{\begin{array}{l}
\cos \varphi \frac{\partial p}{\partial x}=-2 p \operatorname{tg} \varphi\left[\cos \omega \frac{\partial \theta}{\partial x}+(\sin \omega-\sin \varphi) \frac{\partial \theta}{\partial y}\right]+\mathrm{A}[\sin \alpha-\sin \varphi \cos (\omega-\alpha)] \\
\cos \varphi \frac{\partial p}{\partial x}=-2 p \operatorname{tg} \varphi\left[(\sin \omega+\sin \varphi) \frac{\partial \theta}{\partial x}-\cos \omega \frac{\partial 0}{\partial y}\right]+\mathrm{A}[-\cos \alpha-\sin \varphi \sin (\omega-\alpha)]
\end{array}\right.
$$

Elles constituent un système de deux équations aux dérivées partielles en $p$ et $\theta$ que nous allons discuter comme en [2.II.2].

\section{Le problème de Cauchy pour les fonctions $p$ ET $\theta$.}

Cherchons à quelles conditions est possible la détermination de deux fonctions $p(x, y), \theta(x, y)$, solutions de (3), prenant sur un arc $\gamma(\xi, \eta)$ appartenant au massif et constituant tout ou partie de sa frontière, les valeurs $p(\xi, \eta), \theta(\xi, \eta)$ résultant d'une distribution des contraintes le long de $\gamma$. C'est en ceci que consiste le problème de CaUCHY pour les équations (3). Les équations (3) écrites pour $x=\xi, y=n$, et les relations :

$d p=\frac{\partial p}{\partial \xi} d \xi+\frac{\partial p}{\partial \eta_{1}} d \eta_{1}, d 0=\frac{\partial 0}{\partial \xi} d \xi+\frac{\partial \eta}{\partial \eta_{1}} d \eta$, forment, par rapport à :

$$
\frac{\partial p}{\partial \xi}, \frac{\partial p}{\partial \eta}, \frac{\partial \theta}{\partial \xi}, \frac{\partial \theta}{\partial \eta},
$$

un système linéaire de quatre équations, dont le déterminant s'annule avec :

$\mathrm{D}=p(\cos \theta d \xi+\sin \theta d \eta)$

$$
[\sin (\theta-\varphi) d \xi-\cos (0-\varphi) d \eta]
$$

Si $D \neq 0$, la résolution est possible sur $\gamma$. Par différentiations successives, on peut alors calculer les dérivées de $p$ et $\theta$ et construire les développements de ces fonctions au voisinage de $\gamma$.

L'annulation de D, entraînant une impossibilité ou une indétermination pour la résolution en cause, intervient dans les circonstances suivantes : 
a) L'arc $\gamma$, le long duquel s'évanouissent les contraintes, constitue la surface libre du massif : $p=0$. Soit $i(\xi, \eta)$ sa pente, on a :

$$
\frac{\partial p}{\partial \xi} \cos i+\frac{\partial p}{\partial \eta} \sin i=0,
$$

et compte tenu des dérivées partielles de $p$ déduites du système (3) $x=\xi, y=\eta, p=0$, s'écrit:

$$
\begin{aligned}
& \cos \varphi \frac{\partial p}{\partial x}=\mathrm{A}[\sin \alpha-\sin \varphi \cos (\omega-\alpha)] \\
& \cos \varphi \frac{\partial p}{\partial y}=-\mathrm{A}[\cos \alpha+\sin \varphi \sin (\omega-\alpha)]
\end{aligned}
$$

cette expression devient :

$$
\sin (\alpha-i)-\sin \varphi \cos (\omega-\alpha-i)=0
$$

Elle détermine $\omega$ (ou $\theta$ ), c'est-à-dire l'orientation des lignes de glissement, qui ne sauraient être choisies arbitrairement au voisinage de la surface libre dont l'inclinaison $i$ satisfait en outre à la condition de possibilité :

$$
|\sin (i-\alpha)|<\sin \varphi
$$

b) L'arc y se confond avec une ligne de glissement. Si celle-ci est de première espèce $\left(\Gamma_{1}\right)$ :

$$
\cos \theta \quad d \xi+\sin \theta \quad d \eta=0
$$

Si c'est une ligne de seconde espèce $\left(\Gamma_{2}\right)$ :

$$
\sin (\theta-\varphi) d \xi-\cos (\theta-\varphi) d \eta=0
$$

Dans l'un et l'autre cas, $D=0$. On en conclut que la résolution du problème de CAUCHY est généralement impossible à partir de données arbitraires $p(\xi, \eta), \theta(\xi, \eta)$ relatives à une ligne de glissement quelconque du massif. D'ailleurs en- tre les données $p$ et $\theta$ existe nécessairement, le long de chaque ligne de glissement, une relation différentielle traduisant l'équilibre du quadrilatère infinitésimal de côtés $d s_{1}, d s_{2}$, déterminé par l'intersection de deux lignes $\left(\Gamma_{1}\right)$ et de deux lignes $\left(\Gamma_{2}\right)$ infiniment voisines; soit, pour chaque ligne de première et de seconde espèce successivement :

$$
\begin{gathered}
d p-2 p \operatorname{tg} \varphi d \theta-\mathrm{A} \cos (\alpha-\theta+\varphi) d s_{1}=0 \\
d p+2 p \operatorname{tg} \varphi d \theta-\mathrm{A} \sin (\alpha-\theta) d s_{2}=0
\end{gathered}
$$

De l'existence des conditions de possibilité (6), jouant pour les sols pulvérulents le rôle des équations (2.II.7,8) propres aux sols cohérents, on conclut à une organisation identique des massifs de chaque espèce en équilibre limite, à l'inclinaison près des lignes de glissement, orthogonales dans le premier cas, et formant entre elles l'angle $(\pi / 2)-\varphi$ (fig. 55) dans le second. Mais contrairement aux relations caractéristiques des vases, les relations caractéristiques (6) des sables ne sont intégrables que dans des circonstances très limitées, notamment pour $\mathrm{A}=0$, ce qui reviendrait à négliger les forces de volumes, éventualité que nous excluons et aussi dans le cas d'un équilibre parallèle à un champ de filtration uniforme dont nous laissons le lecteur poursuivre l'examen par un processus analogue à celui mis en œuvre à propos des sols cohérents [2.II.5].

\section{Equilibre de Raninine.}

Un équilibre de Rankine est caractérisé par la constance de $\theta$ dans tout le massif dont les lignes de glissement forment alors un réseau de droites. A titre d'application, cherchons à quelle condition la présence d'un champ de filtration ne met pas obstacle à l'existence d'un tel ćtat.

Compte tenu de la constance recherchée de $\theta$, les équations (3) donnent.

$$
\begin{aligned}
& \cos \varphi \frac{\partial p}{\partial x}=-\Phi \frac{\partial \Phi}{\partial x}(1-\sin \varphi \sin \alpha)-\left(\delta+\bar{\omega} \frac{\partial \Phi}{\partial y}\right) \cos \alpha \sin \varphi \\
& \cos \varphi \frac{\partial p}{\partial y}=-\left(\delta+\bar{\omega} \frac{\partial \Phi}{\partial y}\right)(1+\sin \varphi \sin \alpha)-\bar{\omega} \frac{\partial \Phi}{\partial x} \cos \alpha \sin \varphi
\end{aligned}
$$

dont la compatibilité $\frac{\partial^{2} p}{\partial x \partial y}=\frac{\partial^{2} p}{\partial y \partial x}$ entraîne en

posant $: B=\sec \alpha \operatorname{cosec} \varphi$ :

$$
\frac{\partial^{2} \Phi}{\partial x^{2}}-2 \mathrm{~B} \frac{\partial^{2} \Phi}{\partial x \partial y}-\frac{\partial^{2} \Phi}{\partial y^{2}}=0
$$

En outre, et pour tout écoulement permanent, $\Phi$ satisfait à l'équation de continuité :

$$
\frac{\partial^{2} \Phi}{\partial x^{2}}+\frac{\partial^{2} \Phi}{\partial y^{2}}=0
$$

Nous sommes ainsi amenés à résoudre le système complètement intégrable : 
$\frac{\partial^{2} \Phi}{\partial x^{2}}-\mathrm{B} \frac{\partial^{2} \Phi}{\partial x \partial y}=0 . \frac{\partial^{2} \Phi}{\partial y^{2}}+\mathrm{B} \frac{\partial^{2} \Phi}{\partial x \partial y}=0$

Son intégration donne :

$\frac{\partial \Phi}{\partial x}-\mathrm{B} \frac{\partial \Phi}{\partial y}=f(y), \frac{\partial \Phi}{\partial y}+\mathrm{B} \frac{\partial \Phi}{\partial x}=g(x)$.

D'ailleurs $f^{\prime} x=g^{\prime}(x)=\mathrm{C}, f$ et $g$ sont des fonctions linéaires.

En résolvant en $\partial \Phi / \partial x$ et $\partial \Phi / \partial y$, il vient :

$$
\begin{aligned}
& \left(1+\mathrm{B}^{2}\right) \frac{\partial \Phi}{\partial x}=(\mathrm{C} y+\mathrm{D})+\mathrm{B}(\mathrm{C} x+\mathrm{E}) \\
& \left(1+\mathrm{B}^{2}\right) \frac{\partial \Phi}{\partial y}=-\mathrm{B}(\mathrm{C} y+\mathrm{D})+\mathrm{C} x+\mathbf{E}
\end{aligned}
$$

(C, D, E, constantes arbitraires), d'où :

$$
\begin{array}{r}
\Phi(x, y)=\frac{\mathrm{C}}{1+\mathrm{B}^{2}}\left[\frac{\mathrm{B}}{2}\left(x^{2}-y^{2}\right)+x y\right] \\
+\mathrm{F} x+\mathrm{G} y+\mathrm{H}
\end{array}
$$

(F, G, H, constantes arbitraires).

Pour que l'équilibre de Rankrne soit possible, le potentiel de l'écoulement doit être représentable par une forme quadratique du type (7) pouvant d'ailleurs dégénérer en forme linéaire. Dans cette éventualité, la pression en tout point $(x, y)$ résulte de l'intégration de (3), soit :

$$
\begin{aligned}
p=p_{0}+\frac{A}{\cos \varphi} \int[\cos \omega-\sin \varphi \sin (\alpha-\omega)] d x \\
+[\sin \omega+\sin \varphi \cos (\alpha-\omega)] d y
\end{aligned}
$$

En résumé, l'équilibre de Rankine reste possible à une condition très restrictive, puisqu'il est loisible de définir des régions suffisamment limitées du massif où le potentiel est assimilable sinon à une fonction linéaire (champ uniforme), du moins à une forme quadratique du type précédent. On comparera cette conclusion à celle du paragraphe $\left[2 . I I .4 \mathrm{c} .2^{\circ}\right]$ et l'on notera à ce propos une différence essentielle entre sols pulvérulents et cohérents.

\section{Limitation De l'angle des talus.}

Considérons une digue constituée par un matériau homogène et isotrope à travers laquelle s'effectue un écoulement de filtration permanent (fig. 56). Les lignes de courant issues du parement amont qu'elles quittent normalement, sauf dispositions spéciales propres à abaisser la ligne de saturation AR, aboutissent sur le talus aval avec un angle variable. Au point de résur- gence $\mathrm{R}$, la ligne de saturation est tangente au talus aval. Au-dessous du niveau aval, les lignes de courant sont normales au talus. Entre ces deux niveaux, l'angle des lignes de courant et du talus

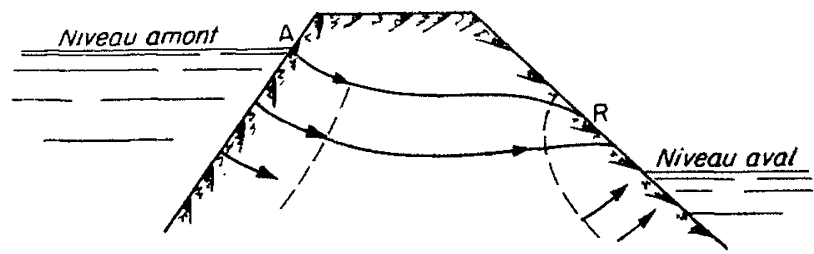

Fig. 56

varie continuemeni de 0 à $\pi / 2$. De ces dispositions résultent divers cas quant à la stabilité des talus en tout état de cause régie par (5).

a) Stabilité du talus aval au voisinage de la résurgence (fig. 57).

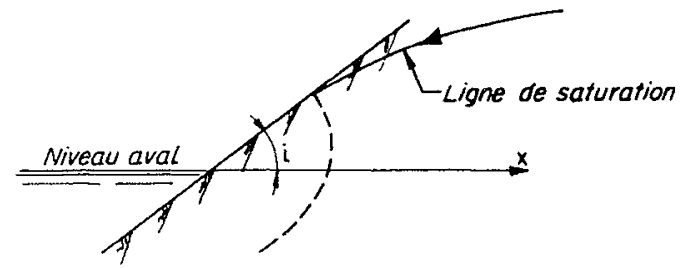

Fig. 57

$A u$ voisinage de la résurgence $R$, où la vitesse de filtration, $\bar{V}=-\mathrm{K} \overline{\text { grad } \Phi}$, et par suite le vecteur $\overline{\operatorname{grad} \Phi}$ sont tangents au talus, on a :

$\frac{d \Phi}{d s}=\frac{d y}{d s}=\sin i, \frac{\partial \Phi}{\partial x}=\sin i \cos i, \frac{\partial \Phi}{\partial y}=\sin ^{2} i$.

$\operatorname{tg} \alpha=-\frac{\mathrm{X}}{\mathrm{Y}}=-\frac{\bar{\omega}(\partial \Phi / \partial x)}{\bar{\omega}(\partial \Phi / \partial y)+\delta}=-\frac{\omega \sin i \cos i}{\omega \sin ^{2} i+\delta}$.

D'autre part, et par (5), la valeur limite de l'inclinaison du talus est :

$$
i=\varphi+\alpha,
$$

d’où :

$$
\operatorname{tg} i=\frac{\delta}{\delta+\pi} \cdot \operatorname{tg} \varphi
$$

6. Stabilité du Talus aval aU-Dessous dU NIVEAU AYAL (fig. 58).

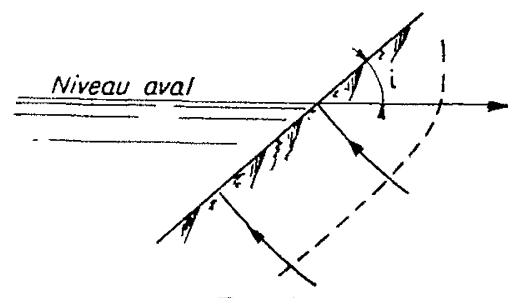

Fig. 58

En tout point du talus situé au-dessous du 
niveau aval, vitesse de filtration $\bar{V}$ et recteur $\widetilde{\operatorname{grad} \Phi}$ sont normaux au talus.

On en déduit :

$\Phi_{x}^{\prime}=\frac{\mathrm{V}}{\mathrm{K}} \sin i, \quad \Phi^{\prime}{ }_{y}=-\frac{\mathrm{V}}{\mathrm{K}} \cos i$,

$$
\operatorname{tg} \alpha=-\frac{\mathrm{X}}{\mathrm{Y}}=-\frac{\lambda \sin i}{1-\lambda \cos i}
$$

avec, comme en : $[2 . \mathrm{II} .5]$ :

$$
\lambda=\frac{\sigma \mathrm{V}}{\mathrm{K}} .
$$

Dès lors, par (5), il vient la relation définissant la pente maximum cherchée:

$$
\operatorname{tg}(0-i)=\frac{\lambda \sin i}{1-\lambda \cos i}
$$

soit encore :

$$
\sin (p-i)=\lambda \sin \varphi
$$

c) Stabilité du talus amont (fig. 59).

La vitesse et le vecteur w grad $\Phi$ sont normaux au talus. Par un calcul analogue à celui développé ci-dessus, il vient :

$\operatorname{tg} \alpha=\frac{\lambda \sin i}{1+\lambda \cos i}$ et $\sin (i-\varphi)=\lambda \sin \varphi$

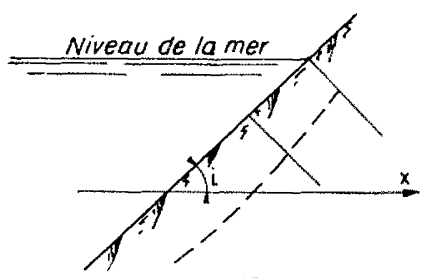

Fis. 5 ?

Les formules (8) (9) (10), qui définissent les pentes limites correspondant aux conditions de stabilité minima des digues, expliquent certains troubles observés lors de la mise en eau des retenues qu'elles bordent. En particulier elles indiquent la possibilité de tenir les talus plus raides à l'amont des massifs qu'à l'aval de ceux-ci.

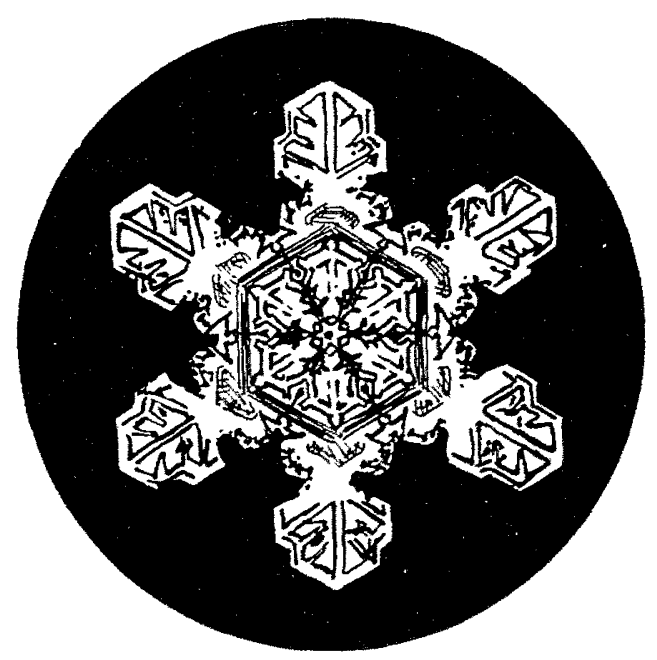

